\title{
Centrifugally driven mass-loss and outbursts of massive stars
}

\author{
Xihui Zhao ${ }^{1,2 \star}$ and Jim Fuller ${ }^{2}$ \\ ${ }^{1}$ Department of Modern Physics, University of Science and Technology of China, Hefei, Anhui 230026, China \\ ${ }^{2}$ TAPIR, Mailcode 350-17, California Institute of Technology, Pasadena, CA 91125, USA
}

Accepted 2020 April 17. Received 2020 April 17; in original form 2020 March 18

\begin{abstract}
Rotation and mass-loss are crucially interlinked properties of massive stars, strongly affecting their evolution and ultimate fate. Massive stars rotating near their break-up limit shed mass centrifugally, creating Be stars with circumstellar discs and possibly driving outbursts. Using the MESA stellar evolution code, we examine the effects of efficient angular momentum transport on the main-sequence and post-main-sequence rotational evolution of massive stars. In rapid rotators, angular momentum transported from the contracting core to the expanding envelope can spin-up the surface layers past the break-up rate, particularly for stars near (or beyond) the end of the main-sequence and in low-metallicity environments. We also demonstrate that centrifugal instabilities could arise in rapidly rotating massive stars, potentially triggering the S Doradus outbursts observed in luminous blue variable stars. Prior mass accretion from a binary companion increases both the likelihood and the intensity of centrifugal mass-loss. We discuss implications for massive stellar evolution, Be stars, and luminous blue variables.
\end{abstract}

Key words: instabilities - stars: evolution-stars: massive-stars: mass-loss-stars: rotation.

\section{INTRODUCTION}

Massive stars lose a significant fraction of their mass over the course of their lives. For single-star models, most of this massloss arises from line-driven winds propelled by photons scattering off absorption lines of iron-group elements (e.g. Castor, Abbott \& Klein 1975; Vink \& Gräfener 2012; Owocki 2014). However, some massive stars also lose mass through outbursts such as eruptions from luminous blue variable (LBV) stars (e.g. Humphreys \& Davidson 1994; Smith 2017). While the physics of line-driven massloss is largely understood, the mechanisms driving the sporadic outbursts of stars like LBVs are not (see Smith 2014; Owocki 2015, for reviews).

Rotation is a crucial ingredient in the evolution of massive stars, greatly affecting the appearance, evolution, and mass-loss rates (see Maeder \& Meynet 2000; Maeder \& Meynet 2012 for reviews). For instance, Be stars rotate near their break-up velocity and are surrounded by a disc of centrifugally expelled matter (see review in Rivinius, Carciofi \& Martayan 2013). In stars near the Eddington limit, the break-up velocity is reduced by radiation force, such that that even modest rotation could potentially significantly increase mass-loss rates (Friend \& Abbott 1986; Langer, García-Segura \& Mac Low 1999; Maeder \& Meynet 2000; Dwarkadas \& Owocki 2002; Aerts, Lamers \& Molenberghs 2004; Gagnier et al. 2019). Rotational effects are especially important in low-metallicity stars with weak line-driven winds where AM loss through winds is less important (Hirschi \& et al. 2006; Georgy et al. 2013). However, many existing studies focus on main-sequence (MS) evolution, and do not incorporate realistic angular momentum (AM) transport in stellar models.

A well-known yet underappreciated aspect of massive stars is that their maximum AM content $J_{c}$ (assuming rigid rotation) decreases strongly as they evolve off the MS. The reason is that massive stars have very large cores and very tenuous envelopes compared to lower mass stars. As massive stars evolve off the MS, the contraction of the core can outweigh the expansion of the envelope, such that the radius of gyration (defined as $\kappa=I / M R^{2}$, where $I$ is the moment of inertia) can decrease by multiple orders of magnitude (Fig. 1). The maximum AM content $J_{c}$ also decreases, such that a star rotating under break-up on the MS may find itself rotating faster than breakup as it evolves off the MS.

This concept of centrifugally driven mass-loss has been explored (Langer 1998; Maeder \& Meynet 2001; Ekström et al. 2008) but has not received a great deal of attention, perhaps because its operation depends on the AM transport between the core and the envelope. Without AM transport, the envelope will simply expand and spindown, but it can be forced to rotate faster than break-up in the presence of efficient AM transport. Fortunately, asteroseismology of low-mass stars has clearly demonstrated that an efficient AM transport process is at work (e.g. Mosser et al. 2012; Cantiello et al. 2014; Deheuvels et al. 2014; Eggenberger et al. 2017; Van Reeth et al. 2018; Fuller, Piro \& Jermyn 2019), motivating new investigations into the rotational evolution of massive stars.

In this paper, we explore the consequences of centrifugally driven mass-loss in stars evolving off the MS, employing AM transport that roughly matches observations of low-mass stars (Fuller et al. 2019, though see also Eggenberger et al. 2019). In many cases, ordinary 


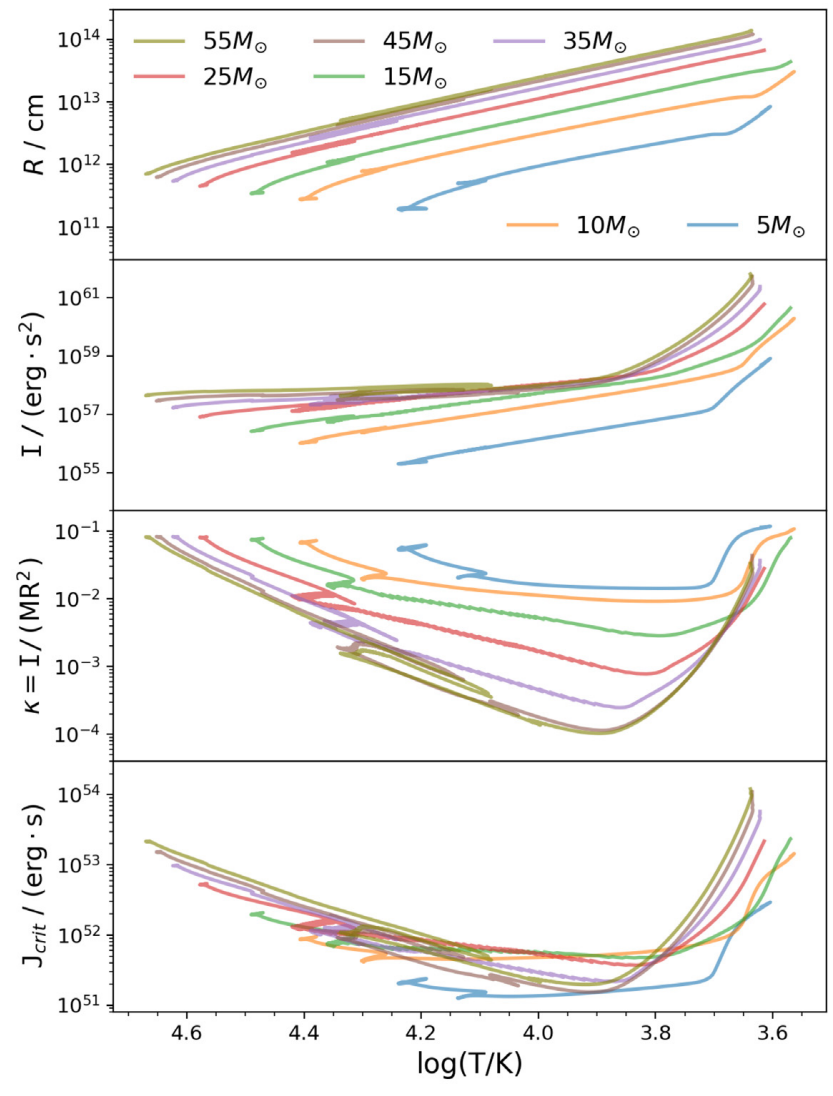

Figure 1. Evolution of stellar structure variables related to the star's rotation rate, as a function of surface temperatures, as stars evolve from the main sequence to core helium burning. Panel 1 (top): The stellar radius, $R$. Panel 2: The moment of inertia, I. Panel 3: The radius of gyration, $\kappa=I /\left(M R^{2}\right)$. Panel 4 (bottom): Maximum angular momentum content of a rigidly rotating star, $J_{\text {crit }}=I \Omega_{\text {crit }}$.

stellar winds remove too much AM during the MS for centrifugally driven mass-loss to occur. However, centrifugal mass-loss can be very important for moderate-mass and low-metallicity stars with lower mass-loss rates. It can also be crucial for stars that have been spun up via accreted mass and AM from a companion star. Furthermore, we begin a preliminary investigation of centrifugally driven instabilities that may drive mass-loss through outbursts rather than gradual equatorial or wind mass-loss. We discuss implications for stellar evolution, Be stars, and LBVs.

\section{ROTATIONALLY DRIVEN MASS-LOSS}

\subsection{Effects of stellar evolution}

As stars evolve, their cores typically contract and decrease their moment of inertia, while their envelopes expand and increase their moment of inertia. In the presence of efficient angular momentum (AM) coupling, the surface rotation $\Omega_{\text {surf }}$ is then determined by the evolution of the star's total moment of inertia $I$ via $\Omega_{\text {surf }}=J / I$, where $J$ is the star's AM. Simultaneously, the star's centrifugal breakup rotation rate scales as $\Omega_{\text {crit }} \sim \sqrt{G M / R^{3}}$ and decreases rapidly as the star's radius $R$ increases. Hence, if the star's mass and AM are conserved, its surface rotation rate can eventually exceed the rotational break-up rate, and the star will be forced to centrifugally expel mass. In later sections, we will account for complications arising from non-rigid rotation, radiation pressure, and mass/AM loss via stellar winds.

Fig. 1 illustrates the evolution of stellar properties that determine how their rotation rates change, for several massive star models. We see that stellar radii nearly always increase, while their temperatures decrease, as they evolve through the main sequence, and then across the Hertzsprung-Russell (HR) diagram as they expand into heliumburning red supergiants. The only exception is the small hook at core hydrogen exhaustion, typically at $\log \left(T_{\text {eff }}\right) \approx 4.3$ for these models. However, we see that the star's moment of inertia $I$ does not always increase strongly, because the expansion of the surface layers is partially compensated by the contraction of the core. This is especially evident for the most massive stars, where the core has a much larger extent on the MS (in both mass and radius), and therefore has a larger influence on the rotational evolution. To demonstrate this, the third panel plots the radius of gyration $\kappa=$ $I /\left(M R^{2}\right)$, which decreases sharply as stars evolve off the MS and become increasingly centrally concentrated.

Consequently, the star's spin rate $\Omega$, which is inversely proportional to $I$ for a rigidly rotating body, can decrease much slower than the break-up limit, such that stars reach break-up and lose mass. We can determine when this occurs by computing the star's maximum AM content $J_{\text {crit }}=I \Omega_{\text {crit }}$, shown in the bottom panel of Fig. 1 . The value of $J_{\text {crit }}$ typically decreases by 1-2 orders of magnitude as massive stars cross the HR diagram, such that they can easily reach their break-up limit, $J>J_{\text {crit }}$, as they evolve off the MS, simply due to their structural evolution. Because of the large change in $J_{\text {crit }}$, this can naturally occur for stars rotating far below their break-up limits on the MS. Again, note that $J_{\text {crit }}$ drops faster for more massive stars, suggesting that centrifugally driven mass-loss could be more common and more intense for more massive stars.

The estimates above assume the star can maintain rigid rotation, which is likely not a good assumption for post-MS stars. In our computations below, in order to compute stars' surface rotation rates, we use asteroseismically calibrated AM transport prescriptions that allow for differential rotation. These calculations thus allow for more realistic predictions of centrifugally driven mass-loss rates.

\subsection{Centrifugal mass-loss rate}

At the surface of a rotating star, mass-loss happens when the centrifugal acceleration $g_{\text {rot }}$ and effective gravitational acceleration $g_{\text {eff }}$ are balanced, which implies the surface rotation rate $\Omega_{\text {surf }}$ reaches the break-up limit $\Omega_{\text {crit }}$. Due to the centrifugal acceleration, the star then ejects mass that carries away AM to keep the surface rotation rate at the break-up limit. To account for this effect, prior works (e.g. Langer 1998; Maeder \& Meynet 2000) typically increase the wind mass-loss rate when $\Omega_{\text {surf }}$ approaches $\Omega_{\text {crit }}$. However, the magnitude of this effect is not clear because it depends on the latitudinal surface temperature and wind driving, and some work (Owocki, Cranmer \& Gayley 1998; Müller \& Vink 2014) suggests the wind mass-loss rate may actually decrease. We instead choose to estimate the centrifugal mass-loss rate purely from the rate at which AM is transported to the surface layers, causing them to be ejected. We note that similar procedures have been performed in Georgy et al. (2013) and Granada et al. (2013).

Assuming that the star rotates rigidly with $J=J_{\text {crit }}=I \Omega_{\text {crit }} \propto$ $\kappa M^{3 / 2} R^{1 / 2}$, the change in AM per unit time is

$\frac{\dot{J}}{J}=\frac{\dot{\kappa}}{\kappa}+\frac{3}{2} \frac{\dot{M}}{M}+\frac{1}{2} \frac{\dot{R}}{R}$. 
Note this relation holds as long as $\Omega_{\text {crit }} \propto \sqrt{M / R^{3}}$. On the other hand, mass-loss carries away AM at a rate

$\dot{J}=\alpha \dot{M} R^{2} \Omega_{\text {crit }}$,

where $\alpha$ accounts for the specific AM of the mass that is lost, in units of $R^{2} \Omega_{\text {surf }}$. For equatorial mass-loss, $\alpha=1$, while $\alpha=2 / 3$ for spherically averaged mass-loss. Setting equations (1) and (2) equal, we have the expression for mass-loss rate:

$\frac{\dot{M}}{M}=\left(\frac{\alpha}{\kappa}-\frac{3}{2}\right)^{-1}\left(\frac{\dot{\kappa}}{\kappa}+\frac{1}{2} \frac{\dot{R}}{R}\right)$.

In massive stars, $\kappa \ll 1$ (see Fig. 1 ), so the first term in parentheses dominates, and

$\frac{\dot{M}}{M} \simeq \frac{\kappa}{\alpha}\left(\frac{\dot{\kappa}}{\kappa}+\frac{1}{2} \frac{\dot{R}}{R}\right)$.

We only employ this mass-loss in our models if $\Omega_{\text {surf }}$ approaches $\Omega_{\text {crit. }}$. While this estimate assumes rigid rotation, which is not strictly valid (especially during post-MS evolution), it has the advantage of being independent of the wind mass-loss rate. Equation (3) may not be precise on a given time step, but it allows mass to be lost at a high rate if $\Omega_{\text {surf }}>\Omega_{\text {crit }}$, and at a small rate otherwise. Hence, our method is reliable for computing the long-term average mass-loss rate when the star evolves such that its surface rotation rate tracks the break-up rotation rate.

In principle, it is possible that the effective value of $\alpha$ could be much larger than unity if a star's AM is transported outwards through a circumstellar disc with little corresponding mass-loss. We cannot discount such a possibility, so our mass-loss rates should perhaps be considered upper limits. However, the AM loss still depends on the production of a disc through centrifugal mass-loss, so our predictions for when centrifugal mass-loss occurs will not be strongly affected.

\subsection{The Eddington limit}

In the discussion above, we neglected radiative effects, but the radiative force in fact can be large in a massive star, whose Eddington factor $\Gamma$ is defined as

$\Gamma=\frac{g_{\text {rad }}}{g_{\text {grav }}}=\frac{\kappa_{R} L}{4 \pi c G M}$,

where $L$ is the star's luminosity and $\kappa_{R}$ is the Rosseland mean opacity at the surface. Radiative forces balance the gravitational force when the Eddington factor approaches unity, i.e.

$g_{\text {grav }}-g_{\text {rad }} \rightarrow 0 \quad$ when $\quad \Gamma \rightarrow 1$.

It is therefore necessary to add a new criterion implementing radiative effects to the mass-loss rate, especially when the star is close to the Eddington limit. We shall find below that the centrifugal mass-loss time-scale is much larger than the stellar expansion timescale, so that the change in $\Gamma$ with mass-loss can be neglected when deriving equation (1) and in equation (3).

However, the radiative force is important when defining the value of $\Omega_{\text {crit }}$ above which centrifugal mass-loss occurs. Considering radiative effects, mass-loss generally happens when the total acceleration equals zero in a rotating star:

$g_{\text {tot }}=g_{\text {grav }}-g_{\text {rot }}-g_{\text {rad }}=g_{\text {eff }}-g_{\text {rad }}=0$

By using the von Zeipel theorem (von Zeipel 1924) and its generalization (Zahn 1992; Maeder 1999), Maeder \& Meynet (2000) expressed the total gravity at co-latitude $\theta$ as

$g_{\text {tot }}=g_{\text {eff }}\left[1-\Gamma_{\Omega}(\theta)\right]$,

where $g_{\text {eff }}=g_{\text {rot }}+g_{\text {grav }}$, and $\Gamma_{\Omega}(\theta)$ is the local Eddington ratio defined as

$\Gamma_{\Omega}(\theta)=\frac{\kappa_{R}(\theta) L}{4 \pi c G M\left(1-\frac{\Omega^{2}}{2 \pi G \rho_{m}}\right)}$.

Here, $\rho_{m}$ is the average density of the star, while $\Omega$ and $L$ are evaluated at isobaric surfaces (i.e. we assume that the baroclinicity of the star is very small; Zahn 1992; Maeder 1999; Maeder \& Meynet 2000).

Equation (7) has two roots, one is that given by $g_{\text {eff }}=0$, which corresponds to the classical break-up limit:

$\Omega_{\text {crit }, 1}=\sqrt{\frac{2}{3} \frac{G M}{R^{3}}}$.

The other root $\Omega_{\text {crit, } 2}$ corresponds to the condition $\Gamma_{\Omega}(\theta)=1$, and the lower value of these two roots will dominate since mass-loss will start as soon as the lower critical velocity is reached. Maeder \& Meynet (2000) argued that only for an Eddington factor $\Gamma$ larger than 0.639 , the second root $v_{\text {crit, } 2}$ has a lower value than $v_{\text {crit, } 1}$ and is given by

$v_{\text {crit }, 2}^{2}=\frac{9}{4} v_{\text {crit }, 1}^{2} \frac{1-\Gamma_{\max }}{V^{\prime}} \frac{R_{\mathrm{e}}^{2}}{R_{\mathrm{p}}^{2}}$.

Here, $\Gamma_{\max }$ is the largest value of the Eddington factor over the stellar surface, $R_{\mathrm{e}}$ and $R_{\mathrm{p}}$ are the equatorial and polar radius, respectively, satisfying $R_{\mathrm{e}}=\frac{3}{2} R_{\mathrm{p}}$ at break-up, and $V^{\prime}$ is the ratio of the actual volume of a star to the volume of a sphere of radius $R_{\mathrm{p}}$, i.e. $V^{\prime}=V / \frac{4}{3} \pi R_{p}^{3}$. Maeder \& Meynet (2000) further pointed out that the ratio $v_{\text {crit, } 2} / v_{\text {crit, } 1}=1$ when $\Gamma_{\max }=0.639$ and $v_{\text {crit, } 2} / v_{\text {crit, } 1}=$ 0 when $\Gamma_{\max }=1$. The trend in $v_{\text {crit, } 2} / v_{\text {crit, } 1}$ is approximately a linear decrease above $\Gamma=0.639$. We therefore use the following approximation for the second root:

$\Omega_{\text {crit }, 2}=\left(\frac{1-\Gamma}{0.361}\right) \Omega_{\text {crit }, 1}$ when $\Gamma>0.639$.

Since $\Gamma$ is not strongly by centrifugal mass-loss, both $\Omega_{\text {crit, } 1}$ and $\Omega_{\text {crit, } 2}$ scale as $\Omega_{\text {crit }} \propto \sqrt{M / R^{3}}$, so the mass-loss rate formula (3) still applies for the second critical rotation rate $\Omega_{\text {crit, } 2}$.

While there has been substantial debate regarding the effect of rotation on massive star wind mass-loss rates (e.g. Glatzel 1998; Langer 1998; Owocki et al. 1998; Gagnier et al. 2019; Maeder \& Meynet 2000), we emphasize that our predicted mass-loss rates do not depend directly on this relationship, but only on whether the star can approach the break-up limit. If rotationally enhanced mass-loss of the form advocated by Langer (1998) can prevent nearcritical rotation from being realized, centrifugal mass-loss will not occur. However, in light of recent two-dimensional results (Müller \& Vink 2014; Gagnier et al. 2019), this appears unlikely, since rotation only enhances wind mass-loss very close to the breakup limit.

\section{RESULTS}

\subsection{Models}

Constructing stellar models with the MESA stellar evolution code (Paxton et al. 2011, 2013, 2015, 2018, 2019), we compute the evolution of rotating massive stars with large initial mass ranging from $5 \mathrm{M}_{\odot}$ to $55 \mathrm{M}_{\odot}$. Our models have an initial surface rotational 


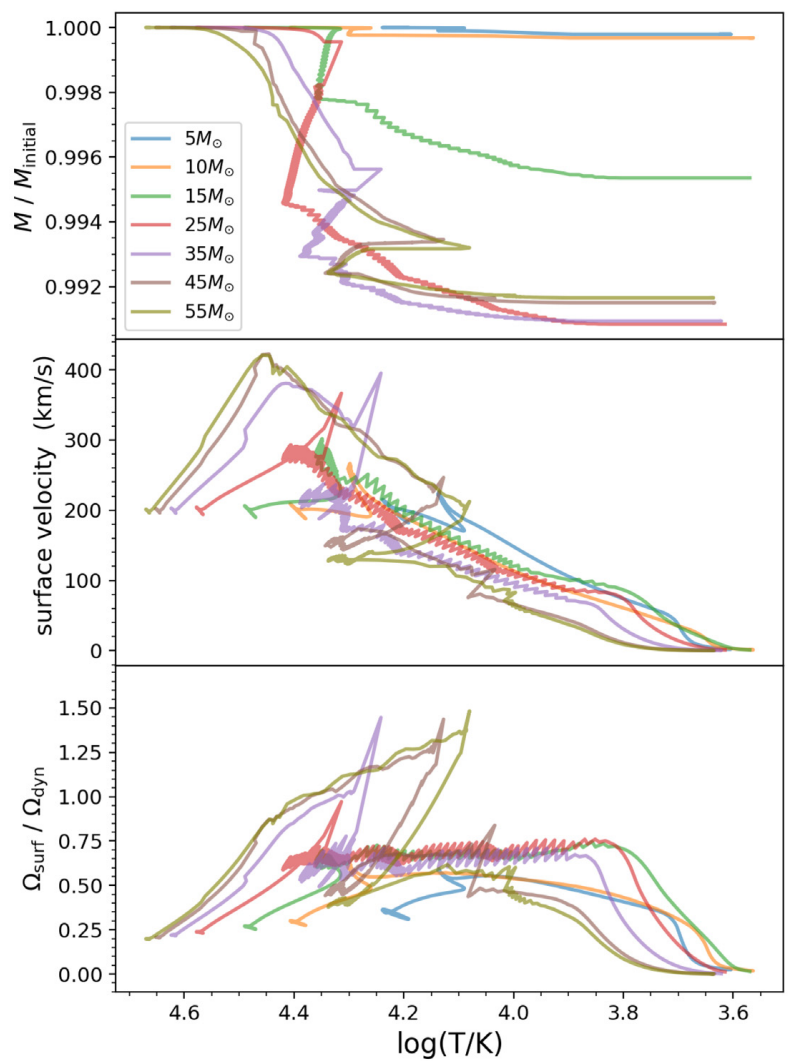

Figure 2. Top: Evolution of the mass of our models including only centrifugal mass-loss and no stellar winds, as a function of their surface temperature. The models begin at the top left and generally evolve towards cooler temperatures. Each model has an initial surface rotational velocity of $200 \mathrm{~km} \mathrm{~s}^{-1}$, with line colour indicating initial mass. Stars lose only a very small fraction of their mass due to centrifugal mass-loss, but most of the mass-loss occurs during the brief phase when stars cross the Hertzsprung gap (see Fig. 3). Middle: Evolution of the surface rotational velocity. Bottom: Dimensionless surface spin rates in units of $\sqrt{G M / R^{3}}$. Note that the dimensionless spin increases to values near unity, at which point centrifugal mass-loss enforces $\Omega / \Omega_{\text {dyn }} \sim \sqrt{2 / 3}$ until further expansion of the star reduces the dimensionless spin.

velocity of $200 \mathrm{~km} \mathrm{~s}^{-1}$, a fairly typical ZAMS value for O/B type stars in the Milky Way and Magellanic clouds (Dufton et al. 2006b; Mokiem et al. 2006; Hunter et al. 2008; Dufton et al. 2013; RamírezAgudelo et al. 2013). We will study stars with solar metallicity $Z=0.017$ and LMC metallicity $z=0.0085$. Rotational mixing is included via Eddington-Sweet circulation with the usual rotational mixing factor of $3.33 \times 10^{-2}$ (see Appendix B for a inlist). The models use the default centrifugal distortion in MESA with limited maximum centrifugal forces, and the AM transport prescription from Fuller et al. (2019).

Our models use MESA's MLT ++ prescription to handle the structure of super-Eddington near-surface layers. The structure of these layers (typically from the iron opacity peak outwards) is dependent on this approximation, as is the radius of the star. Hence, our models do not exhibit the same envelope inflation seen in models without a similar prescription (e.g. Gräfener, Owocki \& Vink 2012). We shall discuss uncertainties associated with this issue in Section 5 .

We include centrifgual mass-loss as discussed in Section 2.3. To summarize, we approximate the surface rotational break-up limit
$\Omega_{\text {crit }}$ to be

$\Omega_{\text {crit }}= \begin{cases}\Omega_{\text {crit }, 1}=\sqrt{\frac{2}{3} \frac{G M}{R^{3}}}, & \Gamma<0.639 \\ \Omega_{\text {crit }, 2}=\left(\frac{1-\Gamma}{0.361}\right) \sqrt{\frac{2}{3} \frac{G M}{R^{3}}}, & \Gamma>0.639\end{cases}$

For surface rotation rates above $\Omega_{\text {crit }}$, we impose a mass-loss rate of

$\dot{M}=M\left(\frac{\alpha}{\kappa}-\frac{3}{2}\right)^{-1}\left(\frac{\dot{\kappa}}{\kappa}+\frac{1}{2} \frac{\dot{R}}{R}\right)$,

with $\alpha=1$. To aid numerical convergence, we smoothly increase mass-loss towards equation (14) as the critical rotation rate is approached, allowing some centrifugal mass-loss at lower rotation rates. We run our models until most of the helium in the core has been burned into carbon and oxygen, i.e. the helium mass fraction in the core is below 10 per cent.

\subsection{Results without wind}

To better understand the effects of stellar evolution and centrifugally driven mass-loss, we first construct models without mass-loss via radiatively driven winds. These models conserve mass and AM, unless they lose mass centrifugally, and are simpler to understand. While real stars do lose mass and AM through winds, our models in this section are similar to very low-metallicity stars, for which mass-loss is irrelevant. They also resemble the post-mass transfer evolution of stars whose AM has been rejuvinated by accretion from a companion.

Fig. 2 displays the evolution of stellar mass and rotation velocity as a function of effective temperature for our stellar models. During most of the MS, centrifugal mass-loss does not occur (or occurs at low levels) because our models are born rotating well below their break-up velocity, and they must evolve substantially before any centrifugal mass-loss can occur. During this stage, the stellar mass is nearly constant as stars evolve to cooler temperatures. Stars with larger initial rotational velocities would lose mass earlier along the MS, and could create Oe/Be stars, though the MS mass-loss rate is typically modest, as shown in Fig. 3.

However, the centrifugal mass-loss rate can increase by orders of magnitude near and just after the TAMS, typically at surface temperatures $\log (T) \approx 4.3-3.9$. It is at this stage of evolution that core contraction is fastest, driving centrifugal mass-loss at rates up to $10^{-4} \mathrm{M}_{\odot} \mathrm{yr}^{-1}$, with higher mass-loss rates for more massive stars. Higher mass models typically lose more total mass than lowmass stars, though none of our models lose more than 1 percent of their mass via centrifugal mass-loss. Mass-loss typically ceases at temperatures of $\log T \lesssim 3.9$, when stars start to develop massive convective envelopes, causing the moment of inertia and $J_{\text {crit }}$ to increase sharply, such that stars no longer rotate above their breakup velocities.

The total amount of centrifugal mass-loss is easily understood, assuming near rigid rotation. The ejected mass carries away an AM content of

$\Delta J=\alpha \Delta M R^{2} \Omega_{\text {crit }}$.

Hence, in order for a star to lose all of its $\mathrm{AM}, J=\kappa M R^{2} \Omega_{\text {crit }}$, and assuming $\alpha \sim 1$ the amount of mass lost is simply

$\frac{\Delta M}{M} \sim \kappa$.

As shown in Fig. 1, $\kappa \sim 10^{-3}-10^{-2}$ when centrifugal mass-loss is occurring, which together with equation (16) accounts for the small 


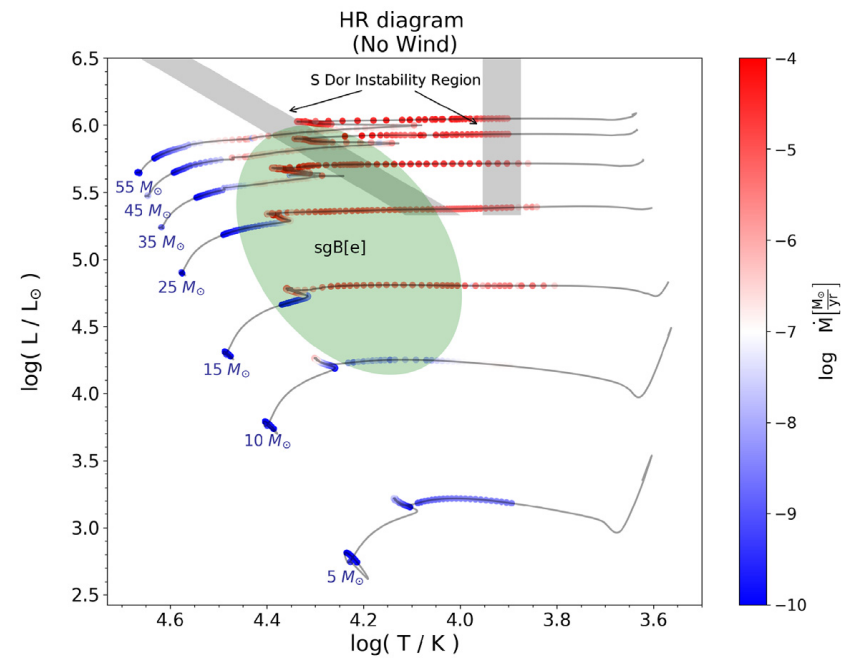

Figure 3. Hertzsprung-Russell diagram showing model evolutionary tracks of stars with an initial surface rotation velocity of $200 \mathrm{~km} \mathrm{~s}^{-1}$, including only centrifugal mass-loss and no winds. Points are coloured by mass-loss rate. Large mass-loss rates are achieved in the Hertzsprung gap, near the empirical location of S Doradus variability cycles (grey regions) and super-giant B[e] stars (green region) as shown in Smith \& Tombleson 2015.

fraction of mass lost. In a real star with differential rotation, the core rotates faster than the surface and the star has more AM, thus more mass can be lost than predicted by equation (16), but our numerical calculations indicate the total mass-loss remains small.

Fig. 2 also shows the evolution of the surface rotation rate of our windless models. For less massive stars $\left(M \lesssim 10 \mathrm{M}_{\odot}\right)$, the core contraction and envelope expansion roughly balance, such the surface rotation velocity is nearly constant. However, in massive stars, the large contracting core overwhelms the expanding envelope, donating enough AM to increase the surface rotation rate of the star towards break-up, even during the MS. Once centrifugal mass-loss begins, the surface velocity tracks the break-up rotation velocity (apart from numerical inaccuracies) until the mass-loss ceases at cooler temperatures where the star's maximum allowable AM content $J_{\mathrm{c}}$ increases. At this point, the surface rotation rate approaches zero, as the star's moment of inertia becomes very large.

\subsection{Results with wind}

Real stars lose mass and AM through line-driven winds, complicating the evolution from the simple scenario above. We examine the coupled effects of centrifugal mass-loss and wind mass-loss using the 'Dutch wind' prescription in our MESA models, with a scaling factor of 0.5 . We use this value because a reduction factor of at least 2-3 from prior estimates, due to wind clumping, is necessary to reproduce observational data (see e.g. Smith 2014; Vink 2017).

Fig. 4 illustrates the evolution of mass and surface rotational velocity of our models with wind mass-loss. Compared to Fig. 2, it is obvious that models with wind lose much more mass, and their surface rotational velocities decrease more quickly. Such differences are especially prominent for stars with larger initial mass because the wind carries away enough AM to prevent the star from ever rotating near break-up. We note the sudden decrease in rotational velocity at $\log (T / \mathrm{K}) \lesssim 4.4$ due to the bistability jump at which the wind mass-loss rate increases sharply. Stars with $M \lesssim 10 \mathrm{M}_{\odot}$ are not strongly affected due to their low wind massloss rates. We note that these models never rotate faster than break-

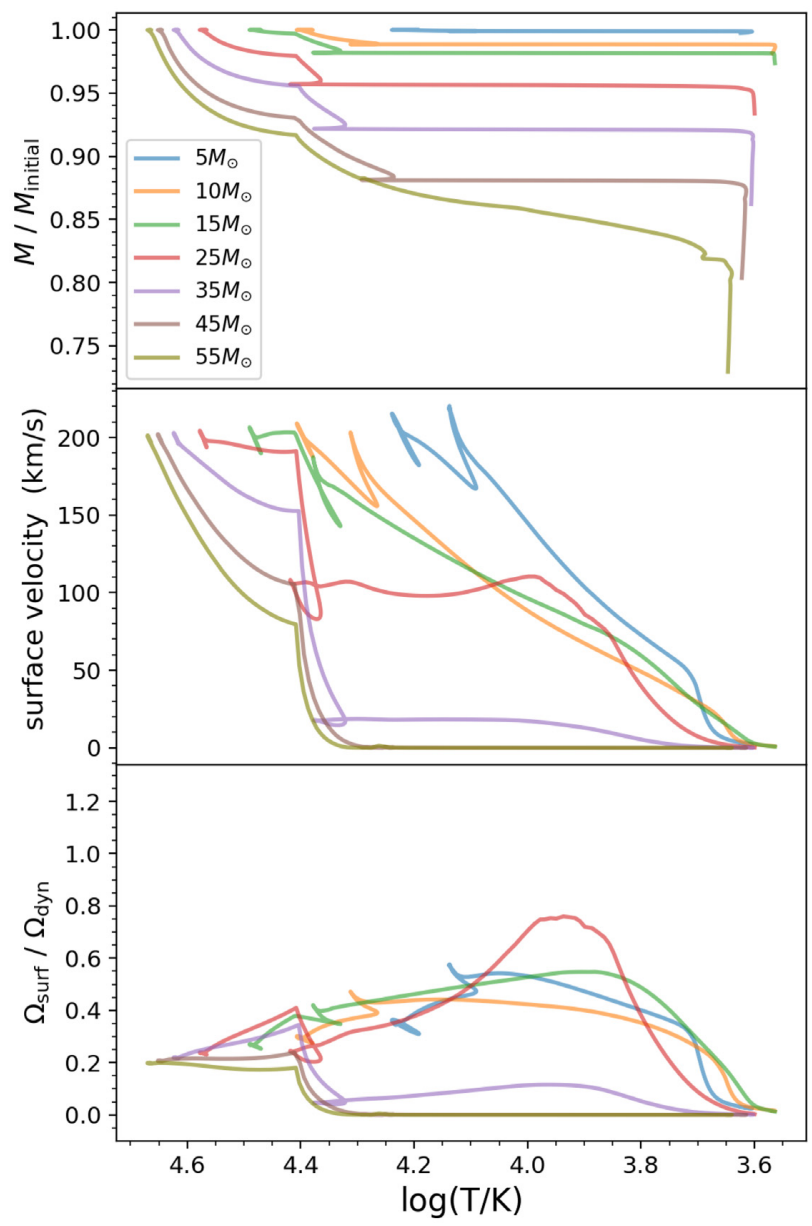

Figure 4. Same as Fig. 2, but for models at solar metallicity with wind mass-loss. These stars generally lose much more mass from winds than from centrifugal mass-loss, causing their surface velocities to decrease such that they typically remain rotating slower than their break-up velocities.

up, yet they still lose mass according to our numerical prescription (Appendix B2), which ramps up the mass-loss smoothly as the star approaches break-up. The actual threshold of $\Omega_{\text {surf }} / \Omega_{\text {dyn }}$ for centrifugal mass-loss in Be stars is still debated, but is likely significantly less than unity (Huang, Gies \& McSwain 2010; Zorec et al. 2016). While our numerical implementation is far from perfect, it is clear that centrifugal mass-loss does begin at subcritical rotation rates and likely intensifies as the break-up limit is approached.

In Fig. 5, we present Hertzsprung-Russell diagrams of stars with centrifugal mass-loss and stellar winds, with points again coloured by mass-loss rate. It is clear that most mass-loss results from stellar winds, while centrifugal mass-loss only accounts for a very small fraction. However, even in these models, the centrifugal mass-loss does become substantial for $\sim 20 \mathrm{M}_{\odot}$ stars crossing the Hertzsprung gap. Centrifugal mass-loss is most prominent in the mass range $\sim 15-25 \mathrm{M}_{\odot}$ because lower mass stars experience less evolutioninduced spin-up, and higher mass stars lose too much AM through winds. While centrifugal mass-loss is negligible compared to the total mass lost, it may cause stellar outbursts in this part of the HR diagram (see Section 4) if centrifugal mass-loss occurs episodically. Interestingly, this mass-loss is largest (and would likely produce more violent outbursts) in stars near the S Doradus instability 


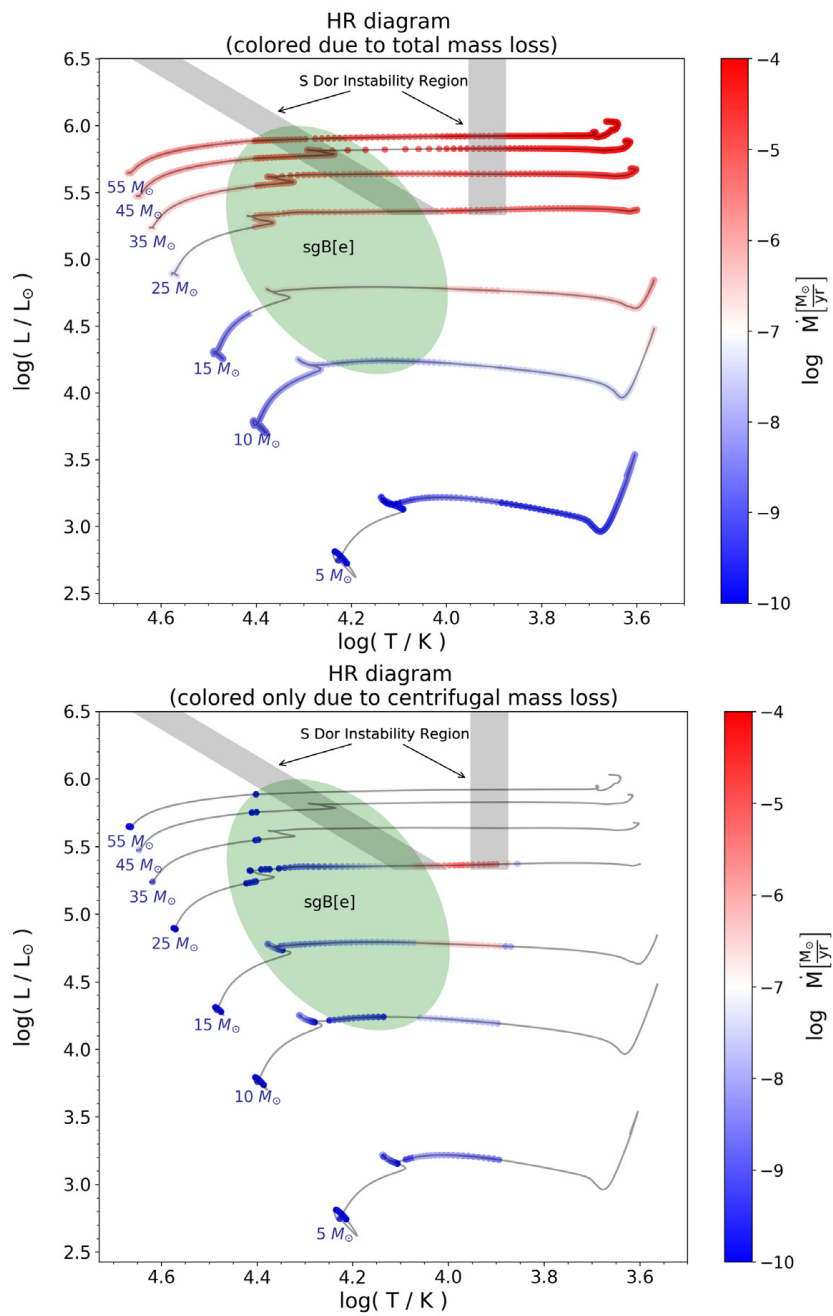

Figure 5. Same as Fig. 3, for models including both centrifugal mass-loss and stellar winds. Top: Points are coloured by total mass-loss rate. Bottom: Points are coloured only by centrifugal mass-loss, and it is evident that centrifugal effects are insignificant, except for some models crossing the Hertzsprung gap.

region, and near the $\mathrm{sgB}[\mathrm{e}]$ stars, both of which are known to exhibit outbursts that eject mass.

\subsection{Effects of metallicity}

Massive star winds are sensitive to stellar metallicity, since they are driven by absorption lines of iron group elements. Hence, we expect lower metallicity stars to lose less mass and AM via linedriven winds, potentially increasing the importance of centrifugal mass-loss. To demonstrate the shifting emphasis from stellar winds to centrifugally driven mass-loss in low-metallicity regions, we construct models with initial metallicity half of the previous ones, i.e. $Z=8.5 \times 10^{-3}$.

Fig. 6 illustrates the evolution of mass and surface rotational velocity of our low-metallicity models, and Fig. 7 shows the centrifugal mass-loss rates. We can see that, compared to solarmetallicity stars, the surface rotation velocities of low-metallicity stars remain larger because winds remove much less AM. The higher AM fuels much larger centrifugal mass-loss rates, especially for high-mass stars. We see high post-MS centrifugal mass-loss

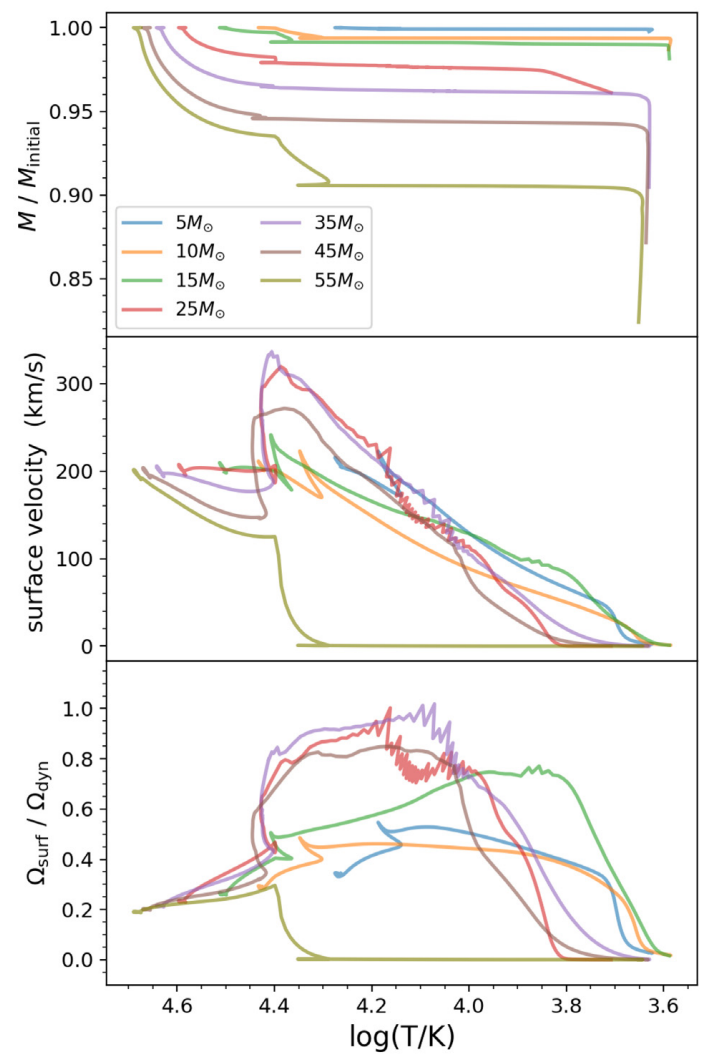

Figure 6. Same as Figs 2 and 4, but for stars with half-solar metallicity $(Z=$ 0.0085). Compared to solar metallicity, the mass-loss rates are significantly lower, allowing the stars to retain more angular momentum, such that centrifugal mass-loss is more pronounced.

extending up to $\approx 45 \mathrm{M}_{\odot}$, compared to only $\approx 25 \mathrm{M}_{\odot}$ for solar metallicity. The centrifugal mass-loss also occurs over a broader span of the HR diagram, but remains largest as stars cross the HR gap, near the locations of $\mathrm{sgB}[\mathrm{e}]$ and $\mathrm{S}$ Doradus stars. While centrifugal mass-loss remains a small fraction of the total massloss, it can dominate the mass-loss rate for post-MS B/A/F-type supergiants evolving into red supergiants.

These results suggest that centrifugal mass-loss should be much more prominent in low-metallicity environments. If the formation of Be stars, $\operatorname{sgB}[\mathrm{e}]$ stars, and LBV outbursts is linked to centrifugal mass-loss, we expect these phenomena to be more common in lowmetallicity galaxies such as the LMC and SMC. For Be stars, this appears to be the case (e.g. Peters et al. 2019). In particular, these single-star models predict more high-mass, high-temperature, and high-luminosity Be stars in low-metallicity environments, whereas the increase for lower mass stars is more mild. While these lower mass stars dominate $\mathrm{Be}$ rates due to the steep initial mass function, the mass sensitivity of centrifugal mass-loss may help distinguish it from binary Be star formation models.

\subsection{Binary interaction}

Centrifugally driven mass-loss may be more pronounced in binary systems where the primary star has accreted mass via stable mass transfer or a stellar merger. The accretion event can replenish the AM of the star, causing it to spin much more rapidly at the end of the MS, intensifying centrifugal mass-loss. 


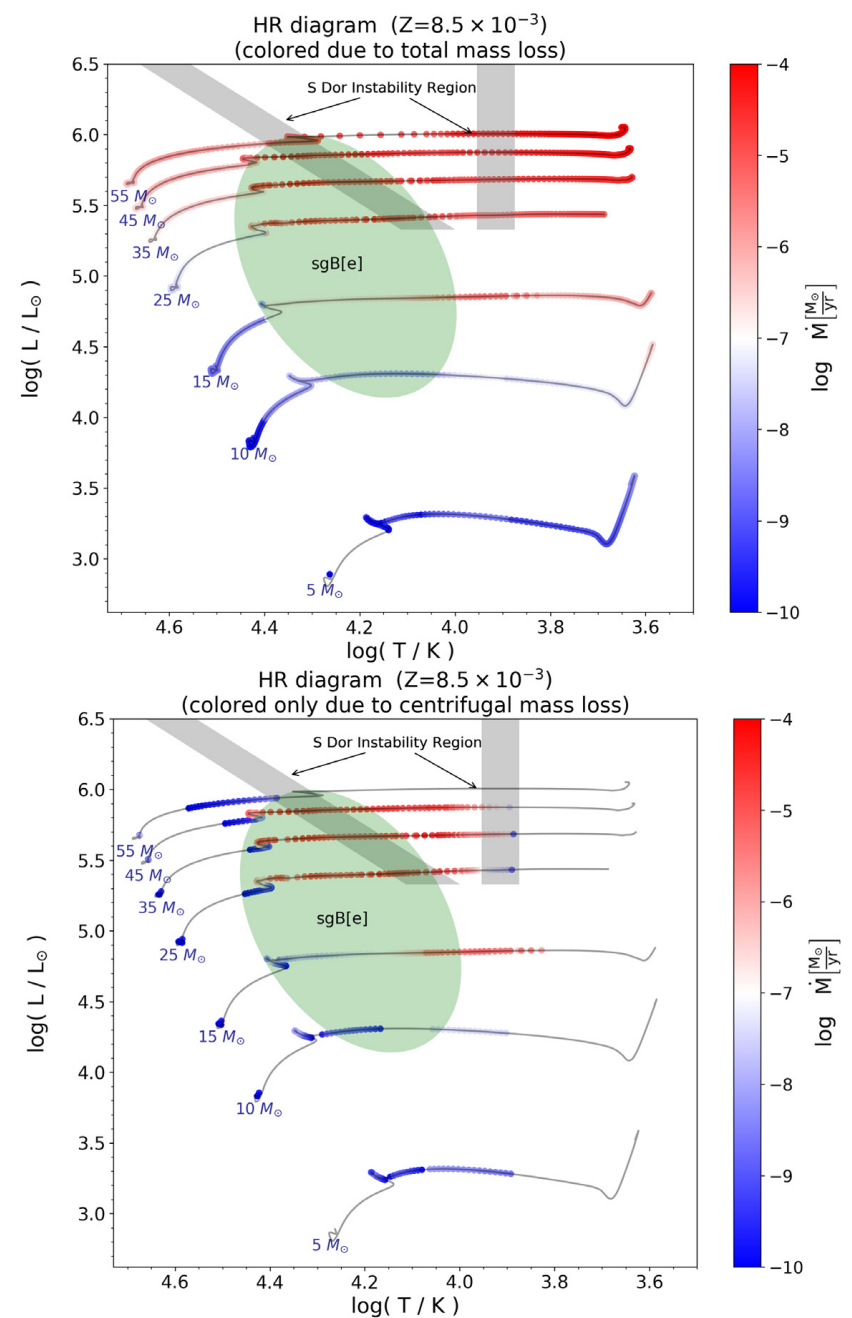

Figure 7. Same as Fig. 5, but for stars with half-solar metallicity. Centrifugal mass-loss is much more common for these models, frequently occurring near the $\mathrm{sgB}[\mathrm{e}]$ and $\mathrm{S}$ Doradus instability regions.

To simulate such a binary scenario in our MESA models, we evolve them until the central hydrogen mass fraction is $X=1 \%$, and then add $10 \mathrm{M}_{\odot}$ using relax_mass. This mimics a stellar merger as the star expands near the end of the MS. After the mass change, we use relax_omega_div_omega_crit to adjust the surface rotation rate until $\Omega_{\text {surf }} / \Omega_{\text {crit }}=1$, which is reasonable because a star need only gain $\sim \kappa M$ in order to spin-up to break-up, and from Fig. 1 we see that typically $\kappa \sim 10^{-2}$ near the end of the MS. We then let the star evolve with both stellar wind and centrifugal mass-loss until the central helium mass fraction is below 10 per cent.

Implementing the aforementioned binary prescriptions, we run models with initial masses of 15,25 , and $35 \mathrm{M}_{\odot}$. Fig. 8 displays the evolution of mass and surface rotational velocity, beginning after the accretion event. In each of these models, the hydrogen-burning core grows and ingests new hydrogen after accretion, so that the star returns to the MS, behaving similar to a MS star of the new mass. However, because much of the core hydrogen has already been burnt, the models spend less time on the MS after accretion, and they lose less AM to winds by the time they evolve off the MS again. Hence, upon leaving the MS, our binary models have greater surface velocities and are closer to the break-up limit, intensifying centrifugal mass-loss.

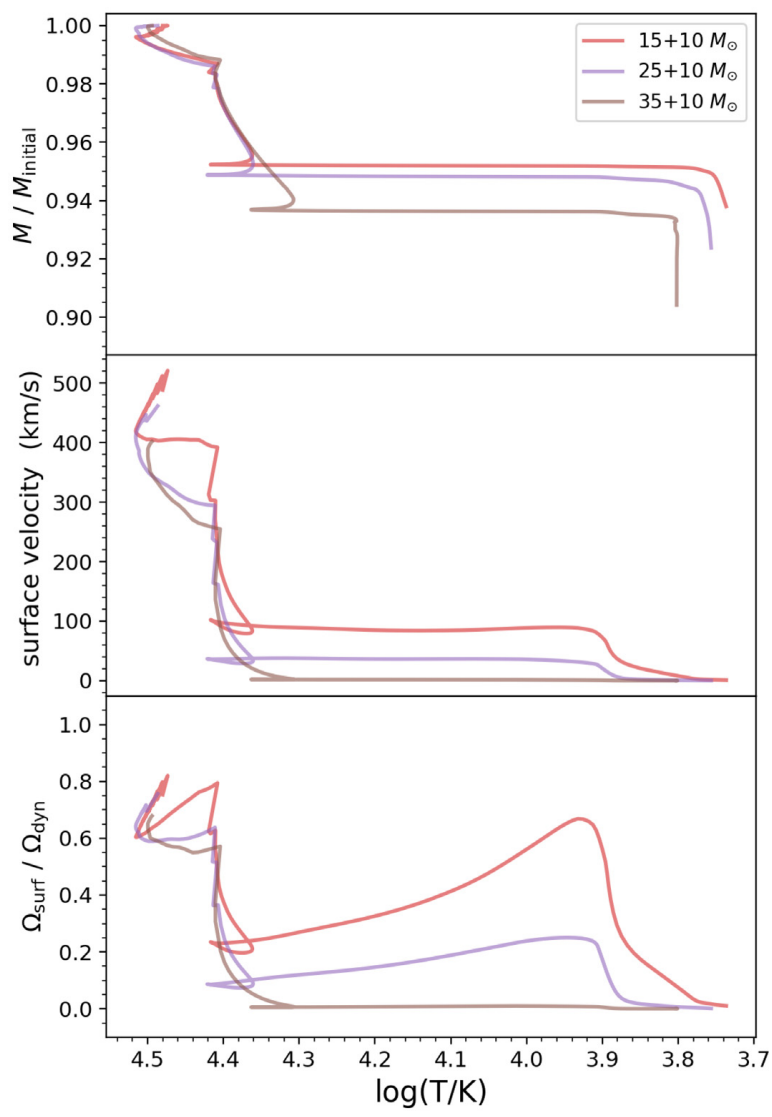

Figure 8. Same as Figs 2, 4, and 6, but for stars that accrete $10 \mathrm{M}_{\odot}$ near the end of the main sequence. Tracks begin at the end of mass accretion. Compared to single stars, the rotation rate is larger at the end of hydrogen burning, intensifying centrifugal effects.

Fig. 9 shows HR diagrams of our binary models. We can see that centrifugal mass-loss rates of $\sim 10^{-5} \mathrm{M}_{\odot} \mathrm{yr}^{-1}$ are achieved for the $15+10 \mathrm{M}_{\odot}$ model as it crosses the Hertzsprung gap, similar to the $25 \mathrm{M}_{\odot}$ model in Fig. 5. The more massive models lose more AM via winds, but the $25+10 \mathrm{M}_{\odot}$ model exhibits more centrifugal massloss than a $35 \mathrm{M}_{\odot}$ single star due to the replenished AM described above. Hence, stellar merger events that occur near the end of the MS can generate more rapidly rotating stars that are more likely to exhibit centrifugally driven outbursts.

Massive stars have small moments of inertia, with $\kappa \sim 10^{-2}$ near the end of the MS (Fig. 1), such that only small amounts of mass accretion can greatly increase their spin. A large increase in the AM of the primary star $\Delta J \sim m J_{\text {crit }} /(\kappa M)$ can be achieved after accreting a small mass $m$, and a $m \sim 1 \mathbf{M}_{\odot}$ merger suffices to spin up the primary to the break-up limit and trigger centrifugal effects. To demonstrate the centrifugal mass-loss generated by ingesting a small companion, we run models with the same ingredients as described above, except that they have initial masses of 25, 35, and $45 \mathrm{M}_{\odot}$ and they gain $1 \mathrm{M}_{\odot}$ during mass transfer.

Fig. 10 displays the evolution of these models. Compared with Figs 4 and 8 , the post-MS ratio $\Omega_{\text {surf }} / \Omega_{\text {dyn }}$ substantially increases, making centrifugal mass-loss much more important. The reason is that ingesting only $1 \mathrm{M}_{\odot}$ spins the primary up to break-up, but it hardly increases the MS lifetime, so the primary is unable to lose much mass through stellar winds after the accretion event. These stars thus enter the post-MS with much more AM, making them 


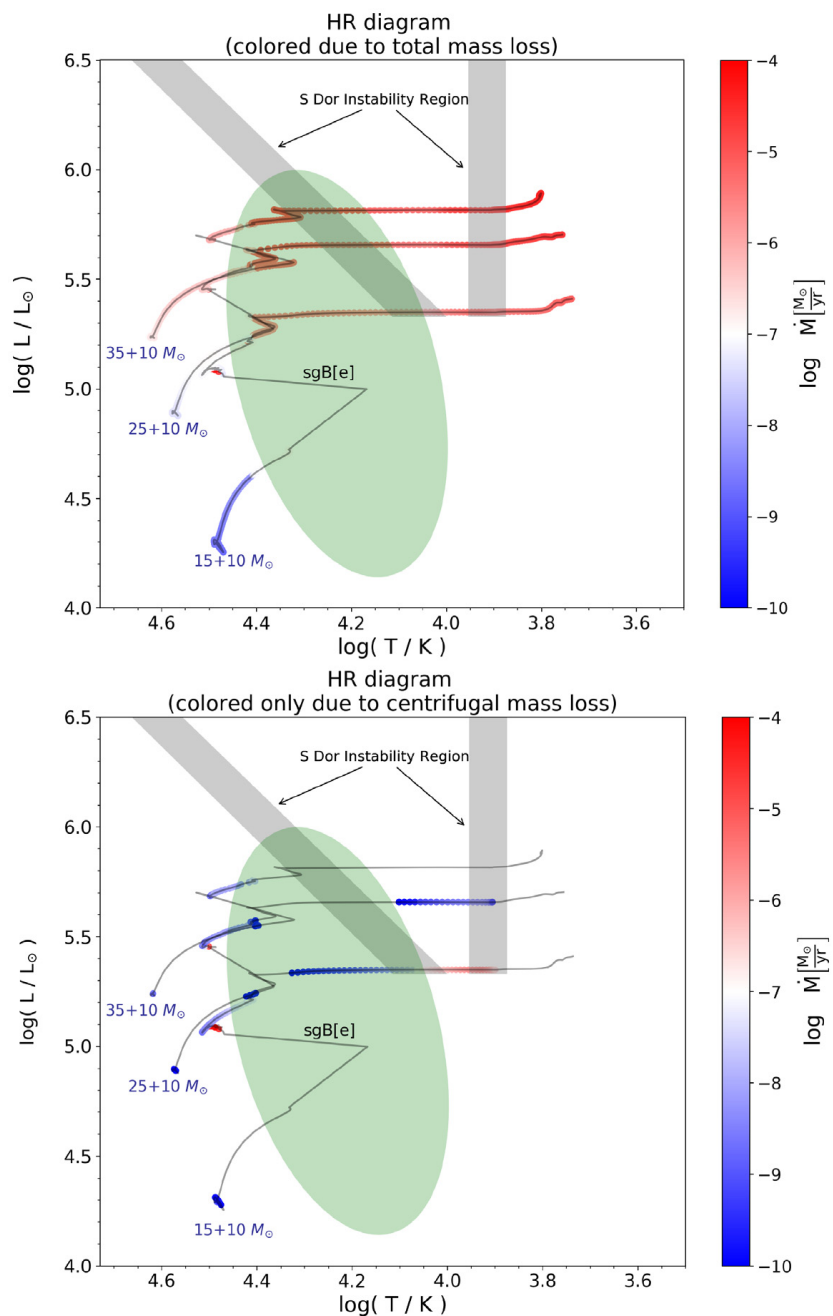

Figure 9. Same as Fig. 5, but for stars that accrete $10 \mathrm{M}_{\odot}$ near the end of the main sequence. Centrifugal mass-loss is slightly more pronounced for these models due to their increased angular momentum when they evolve off the main sequence.

more prone to centrifugal mass-loss when the core contracts and the star crosses the HR diagram.

From the HR diagram in Fig. 11, it is clear that small amounts of mass accretion greatly intensify centrifugal mass-loss rates of all models. The effect is most prominent for the $45 \mathrm{M}_{\odot}$ model, where large centrifugal mass-loss rates of greater than $10^{-5} \mathrm{M}_{\odot} \mathrm{yr}^{-1}$ are achieved within the S Doradus instability region. In contrast, centrifugal effects are nearly negligible when there is no mass gain, due to AM loss by winds (Fig. 5). Hence, assimilating a small companion at the end of MS can fuel vigorous centrifugal massloss during the post-MS, which could play a role in the variability or outbursts of some LBVs.

\section{CENTRIFUGAL INSTAB ILITY}

When centrifugal mass-loss occurs, it could occur steadily, or in sporadic outbursts. To investigate the latter possibility, we perform a stability analysis of perturbations in rotating stars, including the centrifugal force. In our simplified calculation, we focus on adiabatic quasi-radial perturbations. Consider the perturbation of a shell with radius $r$ inside the star, such that the perturbed radial

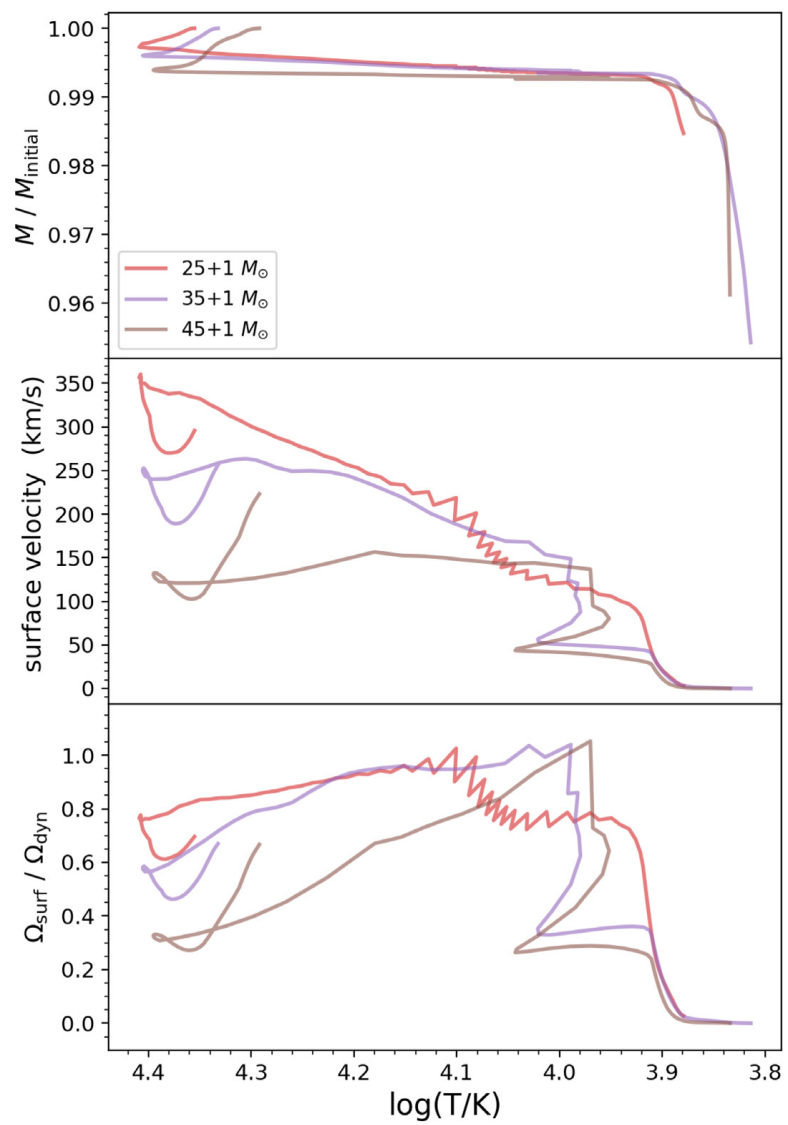

Figure 10. Same as Figs 2, 4, 6, and 8, but for stars that accrete $1 \mathrm{M}_{\odot}$ near the end of the main sequence. Tracks begin at the end of mass accretion. Compared to single stars, the rotation rate is larger at the end of hydrogen burning, intensifying centrifugal effects.

coordinate is

$r^{\prime}=r+\Delta r$

with time dependence

$\Delta r \propto \mathrm{e}^{-i \omega t}$.

Unstable perturbations occur when $\omega^{2}<0$, and the existence of growing modes signals an instability that could result in mass-loss through outbursts.

The Lagrangian perturbation to the specific angular momentum $j=\frac{2}{3} \Omega r^{2}$ is

$\frac{\Delta j}{j}=2 \frac{\Delta r}{r}+\frac{\Delta \Omega}{\Omega}$,

where $\Omega$ is a shellular rotation rate. We now introduce a factor $f_{\Omega}$ describing the change in rotation rate

$\frac{\Delta \Omega}{\Omega}=f_{\Omega} \frac{\Delta r}{r}$.

For conserved specific AM $\Delta j=0$, we have $f_{\Omega}=-2$, while for efficient AM transport during the pulsation, we have $\Delta \Omega=0$ hence $f_{\Omega}=0$. Usually AM is thought to be conserved, but if the plasma is threaded by a magnetic field, for example, magnetic torques could enforce nearly constant rotation and an increased specific AM (at the expense of AM from other layers). The spherically averaged radial component of the centrifugal force over a shell is computed 


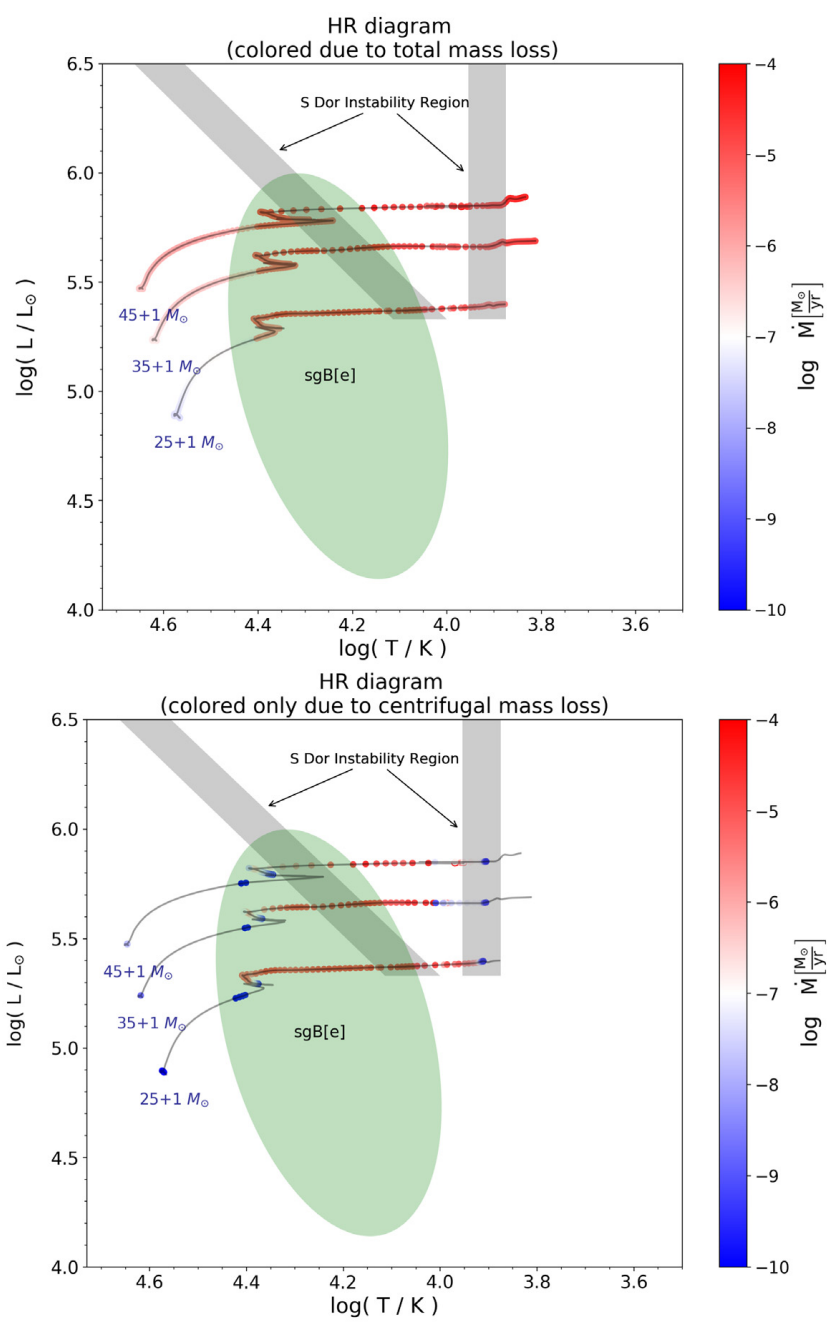

Figure 11. Same as Figs 5 and 9, but for stars that accrete $1 \mathrm{M}_{\odot}$ near the end of the main sequence. Centrifugal mass-loss rates are larger for these models due to their increased angular momentum when they evolve off the main sequence.

as

$$
\begin{aligned}
f_{\text {cen }} & =\frac{1}{4 \pi r^{2}} \int_{0}^{\pi} 2 \pi \Omega^{2} r^{3} \sin ^{3} \theta \mathrm{d} \theta \\
& =\frac{2}{3} \Omega^{2} r .
\end{aligned}
$$

The spherically averaged perturbation to the centrifugal force is then

$\frac{\Delta f_{\text {cen }}}{f_{\text {cen }}}=2 \frac{\Delta \Omega}{\Omega}+\frac{\Delta r}{r}=\left(2 f_{\Omega}+1\right) \frac{\Delta r}{r}$.

Next, we have the perturbed continuity equations

$\Delta \rho+\rho \nabla \cdot \Delta \vec{r}=0$.

For simplicity, we consider homologous perturbations to the stars such that $\Delta r \propto r$, so that

$\frac{\Delta \rho}{\rho}=-3 \frac{\Delta r}{r}$.

This assumption essentially restricts our analysis to modes with long wavelengths in the outer part of the star, i.e. similar to radial fundamental modes in the envelope (though potentially with more complicated dependence in the core). We also assume adiabatic pulsations such that

$$
\frac{\Delta p}{p}=\gamma \frac{\Delta \rho}{\rho}=-3 \gamma \frac{\Delta r}{r} \text {. }
$$

We shall see that instability is most likely to occur near the iron opacity bump in the outer layers of the star. To justify our adiabatic approximation, Fig. 13 shows the thermal time-scale $t_{\text {therm }}=$ $4 \pi r^{2} H \rho \mathrm{C}_{P} T / L$ and dynamical time-scale $t_{\mathrm{dyn}}=H / c_{s}$ near the iron opacity peak, where $H$ is the pressure scale height. The thermal time-scale is typically a few orders of magnitude larger than the dynamical time-scale, indicating that heat cannot diffuse efficiently and an adiabatic approximation is reasonable. Consequently, our analysis does not include strange modes trapped in outermost layers where non-adiabatic growth/damping rates can be very large.

Next, by perturbing the radial Navier-Stokes equation, we have

$$
\begin{aligned}
\rho \frac{\partial}{\partial t} \Delta v_{r}= & -\rho \omega^{2} \Delta r-\frac{\partial}{\partial r} \Delta p \\
& +\frac{\partial p}{\partial r} \frac{\Delta r}{r}-\Delta\left(\rho g_{\text {grav }}\right)+\Delta\left(\rho f_{\text {cen }}\right),
\end{aligned}
$$

where $\Delta v_{r}$ is the perturbed radial velocity, and the background on which we perturb satisfies $v_{r}=0$. Note we have ignored the Coriolis force, which we justify in Appendix A under certain conditions.

The perturbed gravitational force is

$$
\frac{\Delta g_{\text {grav }}}{g_{\text {grav }}}=-2 \frac{\Delta r}{r} \text {. }
$$

To compute $\frac{\partial}{\partial r} \Delta p$, recall in equation (29) we have $\frac{\Delta p}{p} \propto \frac{\Delta r}{r}=$ constant, and

$$
\begin{aligned}
\frac{\partial}{\partial r} \Delta p & =p \frac{\partial}{\partial r}\left(\frac{\Delta p}{p}\right)+\frac{\Delta p}{p} \frac{\partial p}{\partial r} \\
& \approx \frac{\Delta p}{p} \frac{\partial p}{\partial r} .
\end{aligned}
$$

The last line follows from our assumption that $\Delta p / p$ varies slowly, whereas $p$ varies over a pressure scale height that is typically much smaller than $r$ near the iron opacity peak.

To summarize, the relation between $\Delta r$ and other perturbed quantities is

$$
\begin{aligned}
\frac{\Delta r}{r} & =-\frac{1}{3} \frac{\Delta \rho}{\rho}=-\frac{1}{3 \gamma} \frac{\Delta p}{p} \\
& =-\frac{1}{2} \frac{\Delta g_{\text {grav }}}{g_{\text {grav }}}=\frac{1}{2 f_{\Omega}+1} \frac{\Delta f_{\text {cen }}}{f_{\text {cen }}} .
\end{aligned}
$$

From hydrostatic equilibrium, $\frac{\partial p}{\partial r}=-\rho g_{\text {grav }}+f_{\text {cen }}$. Then, plugging in equations (18), (28), and (29) into (26), we finally obtain an expression for $\omega^{2}$ :

$\omega^{2}=\frac{g_{\text {grav }}}{r}\left[3 \gamma-4-\left(2 f_{\Omega}+3 \gamma-1\right) \frac{f_{\text {cen }}}{g_{\text {grav }}}\right]$.

Equation (30) is similar to the classic result for non-rotating stars, which become less stable as $\gamma$ decreases towards $4 / 3$, but with an extra term proportional to the centrifugal force. We note that our local result is very similar to the global result derived in Ledoux (1945) (see also Tassoul 1978), in the limit that AM is conserved $\left(f_{\Omega}=-2\right)$, in which case the centrifugal term is actually stabilizing for $\gamma<5 / 3$ as we expect in massive stars. However, our formula allows for the destabilizing influence of torques that conserve rotation rate instead of $\operatorname{AM}\left(f_{\Omega}=0\right)$, in which case the centrifugal term is destabilizing. In this case, instability occurs even 


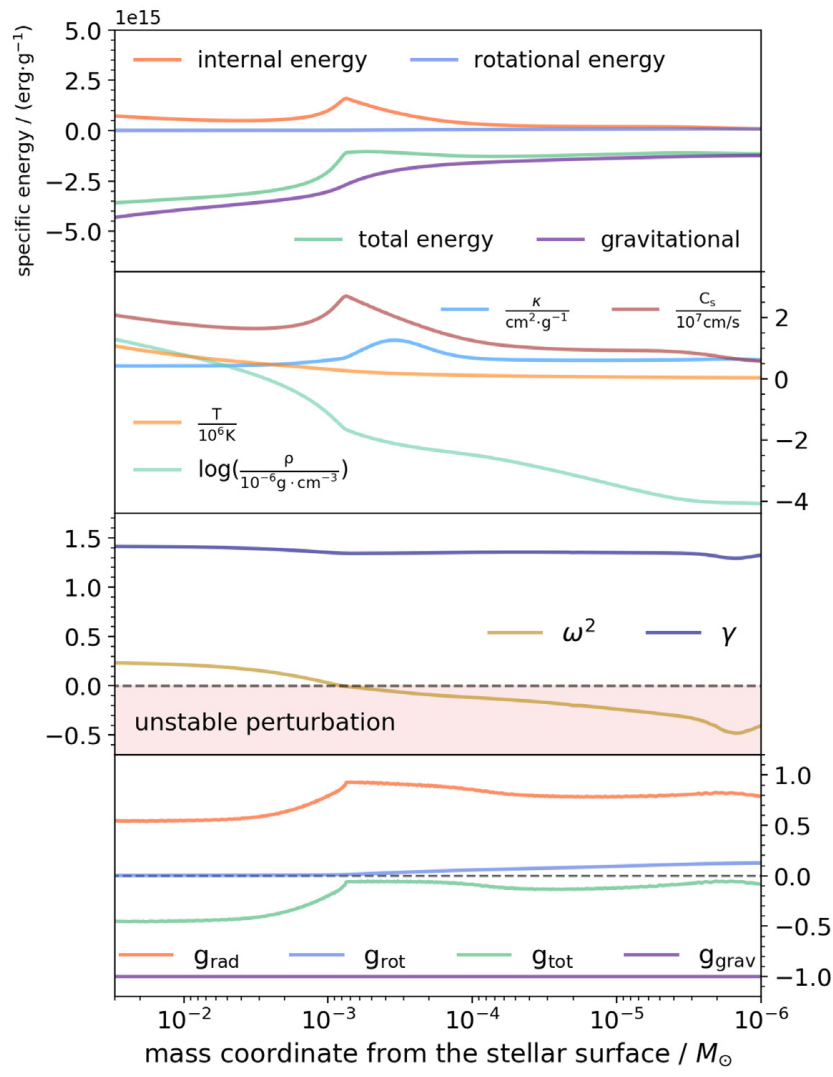

Figure 12. Profiles of stellar properties for a $45 \mathrm{M}_{\odot}$ model when its surface temperature is $T_{\text {eff }}=20000 \mathrm{~K}$. The $x$-axis is the exterior mass, i.e. the mass coordinate measured from the star's surface. Panel 1 (top): Specific energies, with colours corresponding to different kinds of energy as labelled. Panel 2: Opacity, sound speed, density, and temperature in the outer layers of the star. Note the peaks in opacity and sound speed near the iron opacity bump at mass coordinate $\approx 6 \times 10^{-4} \mathrm{M}_{\odot}$. Panel 3: Dimensionless square frequency $\omega^{2}$ of the perturbation in units of $G M / R^{3}$, along with the adiabatic index $\gamma$. Panel 4 (bottom): Specific forces in units of $G M / R^{2}$.

at relatively small values of $f_{\text {cen }} / g_{\text {grav }}$, as small as $f_{\text {cen }} / g_{\text {grav }}=1 / 4$ for $\gamma=5 / 3$, and even smaller values for $\gamma<5 / 3$.

In Fig. 12, we examine the structure of a $45 \mathrm{M}_{\odot}$ star with an effective temperature $T_{\text {eff }}=20000 \mathrm{~K}$. The iron opacity peak occurs at a mass coordinate of $q \approx 4 \times 10^{-4} \mathrm{M}_{\odot}$, and is also associated with a peak in internal energy density, sound speed, and outward radiation force, as well as a dip in $\gamma$. At mass coordinates above this location, we see the local value of $\omega^{2}$ becomes negative, indicating an instability could originate from this location in the star. While the value of $\omega^{2}$ is even more negative closer to the surface of the star, our adiabatic approximation could break down there.

Our perturbation analysis is crude and not suitable for robust predictions. The main point is to highlight the destabilizing influence of the centrifugal force for any perturbation in which AM can be transported rapidly (i.e. shorter than an oscillation time-scale). Since the fundamental mode frequencies of $\gamma=4 / 3$ polytropes approach zero in the absence of rotation, their long pulsation periods will allow for more AM transport during the pulsation cycle, further destabilizing them relative to polytropes of higher $\gamma$. The growth of such a perturbation is fuelled by energy added to the outwardly perturbed fluid element via these (presumably magnetic) torques. Of course, the star must conserve its total AM, so the inner layers must contract (or at least expand by a smaller fraction) in order for

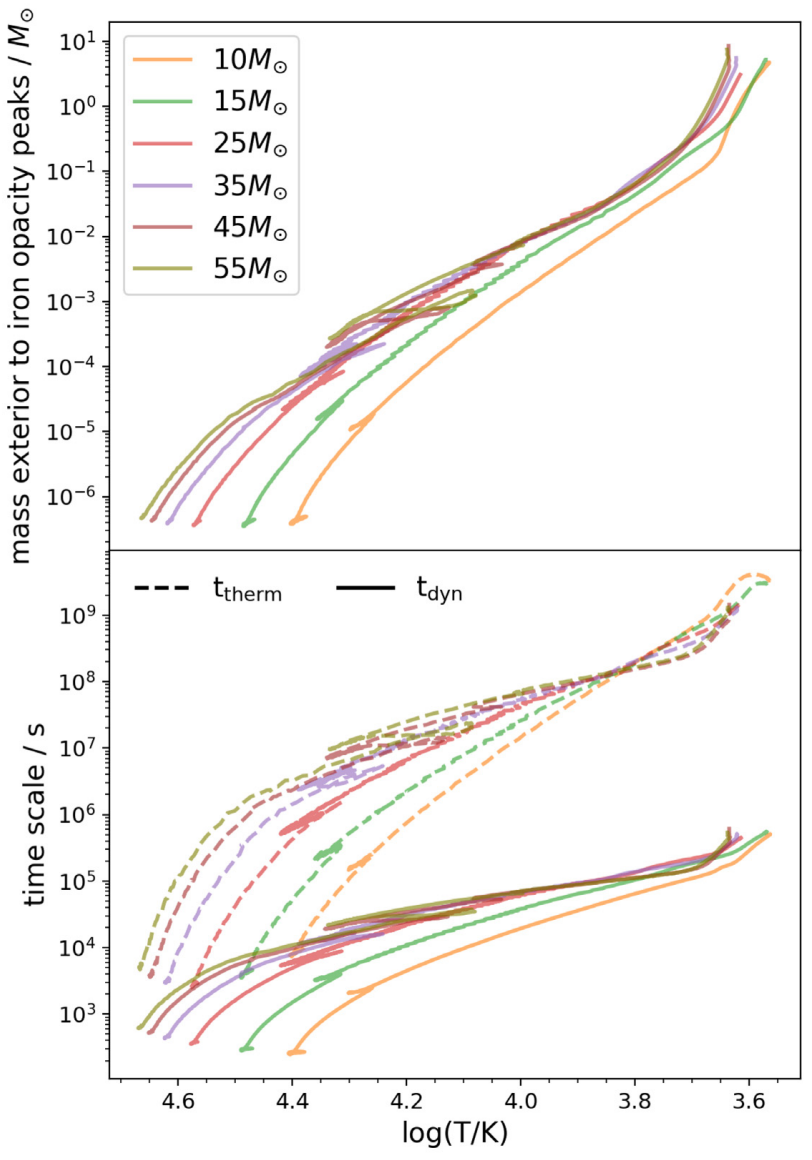

Figure 13. Top: The mass exterior to the iron opacity peak during the evolution of stars with initial masses $15-55 \mathrm{M}_{\odot}$ and initial surface rotation velocity of $200 \mathrm{~km} \mathrm{~s}^{-1}$, as a function of surface temperature. Bottom: Local time-scales at the iron opacity peak, with dashed lines for the thermal timescale $t_{\text {therm }}$ and solid lines for the dynamical time-scale $t_{d y n}$. It is clear that in general $t_{\text {therm }} \gg t_{d y n}$, justifying our adiabatic approximation.

the outer layers to gain energy and AM during the pulsation cycle. Hence, the pulsation mode cannot everywhere have the $\Delta r \propto r$ dependence we have assumed, but must have more complex spatial structure, as we should expect for realistic stars with kinks in their density profiles (Fig. 13).

Crucially, this centrifugal instability does not require superEddington luminosities or near-break-up rotation at the surface of the star, which are the criteria usually examined for massive stars. Instead, the instability is promoted by a low adiabatic index and high rotation rate inside the star, and it feeds on the internal energy and rotational energy of sub-surface layers. The instability is most likely to occur in the outer layers of post-MS massive stars, where $\gamma$ approaches $4 / 3$ over a significant fraction of the stellar radius, unlike some MS models where the iron opacity bump is located at a radius very close to the photosphere. Hence, instabilities causing outbursts are more likely to occur in very luminous stars post-MS such as LBVs, and are less likely to occur in MS stars such as Be stars.

We have performed exploratory one-dimensional hydrodynamic simulations with MESA, including centrifugal forces and AM transport. These simulations do indeed give rise to an instability near the end of the MS, approximately when we find $\omega^{2}<0$ near the iron opacity bump. The instability only occurs in rotating models, but it occurs even at moderate rotation rates well below break- 
up. Hence, we believe the instability is essentially the same as that predicted by equation (30). The outcome can be a steady centrifugally driven wind, or a periodic expansion and contraction of the stellar envelope. While such expansion/contraction does occur in S Doradus outbursts, it typically occurs on a time-scale of $\sim$ weeks in these models, much faster than the slow observed variability of $\mathrm{S}$ Doradus cycles. The instability may saturate when the expanding envelope expands and cools, increasing its adiabatic index and the value of $\omega^{2}$. A detailed investigation requires much more work, but these preliminary numerical calculations (which are global and include non-linear effects) do indicate the presence of a centrifugally driven instability.

If an instability does occur, the outcome is not clear, but it could result in centrifugal ejection of material overlying the iron opacity peak. Fig. 13 plots the mass exterior to the iron opacity peak for stars evolving across the HR diagram. Where centrifugally driven mass-loss is most likely to occur, i.e. $\log T \approx 4-4.3$, the associated mass is $\sim 10^{-5}-10^{-2} \mathrm{M}_{\odot}$. While centrifugal instability may be able to power Be star outbursts and S Doradus outbursts, it likely cannot power giant eruptions from LBVs that eject more than $\approx 1 \mathrm{M}_{\odot}$ of material.

\section{DISCUSSION}

The centrifugal effect included in our models is an important factor in stellar evolution, since many massive stars will reach the break-up limit when they evolve off the MS. We find that such an effect is greater for more massive and lower metallicity stars, especially when they are crossing the Hertzsprung gap. At this stage, centrifugal mass-loss rates as large as $10^{-4} \mathrm{M}_{\odot} \mathrm{yr}^{-1}$ are achieved as shown in Figs 3, 5, and 7. However, for massive stars in high-metallicity regions with strong line-driven winds, centrifugal mass-loss becomes less important because stellar winds carry away most of the star's AM, preventing the star from reaching the breakup limit during the post-MS. Below we discuss implications and caveats of our findings.

\subsection{The Be star phenomenon}

Be stars are known to be very rapidly rotating, often near their breakup velocities (Rivinius et al. 2013), but their formation is not totally understood. Our work suggests that some Be stars can be produced as a result of core contraction and surface rotational acceleration due to efficient AM transport. This single star scenario, proposed by Maeder \& Meynet (2001), also explains the higher abundance of Be stars in slightly aged populations (older than $10 \mathrm{Myr}$ ) and stars near the TAMS (Keller, Bessell \& Da Costa 2000; Aidelman et al. 2018), because more envelope spin-up occurs as stars age and evolve toward the TAMS. Additionally, the larger abundance of Be stars in low-metallicity environments (Peters et al. 2019 and references therein) is naturally explained by the smaller mass/AM loss through winds (rather than having to appeal to larger rotation rates at birth), allowing more stars to reach their breakup rotation rate.

Ekström et al. (2008) predicted the number of stars that reach break-up via this channel during the MS and then compare with the observed fraction of Be stars in clusters at various metallicities. However, they underpredict the observed fraction of Be stars by a factor of a few. While a few effects could account for this discrepancy, an important difference is that their AM transport prescription only accounts for Eddington-Sweet circulation and hydrodynamic turbulence. Consequently, their models (and those in most papers by the Geneva group) exhibit significant differential rotation on the MS, with surface rotation reduced by inefficient AM transport from core to surface. Ekström et al. (2008) agrees that inclusion of magnetic torques via the Tayler-Spruit (TS) dynamo (Spruit 2002) would increase AM transport and Be star formation. In the late stages of preparation of this manuscript, Hastings, Wang \& Langer (2019) examined Be star formation in models including AM transport via the TS dynamo. They indeed find a higher rate of Be star formation, approximately consistent with observations if $\mathrm{Be}$ star are formed when $v_{\text {rot }} / v_{\text {crit }} \gtrsim 0.75$.

Since we now know that AM transport in radiative regions of stars is even more efficient than the TS dynamo predicts (Cantiello et al. 2014), a larger fraction of Be stars should be produced. Because our AM transport prescription and the TS dynamo predict nearly rigid rotation during the MS, we expect to find similar results to Hastings et al. (2019) for MS stars, though we have not performed a full population synthesis calculation. Like Hastings et al. (2019), we find a 'goldilocks' range of $\approx 15-25 \mathrm{M}_{\odot}$ (at solar metallicity) where Be star formation is most efficient, the lower bound arising from a smaller core mass and the upper bound from larger wind AM losses. The upper bound extends to larger mass/luminosity in lowmetallicity environments. If AM is transported in radiative zones via fossil fields (Kissin \& Thompson 2015), AM transport is even more efficient than our estimates, further promoting Be star formation. Our results show that Be formation is most likely to occur in stars just beyond the MS and could also contribute to the population of B[e] supergiants (Kraus 2019). However, because stars evolve across the HR gap extremely quickly, post-MS Be stars will likely be outnumbered by MS Be stars.

Some aspects of Be stars remain difficult to explain with our single star models. For instance, like Hastings et al. (2019), our single star models predict that Be stars are much more common near the TAMS, whereas only a mild increase is observed. Additionally, we may expect higher levels of nitrogen enrichment than observed. Furthermore, we cannot readily explain the higher incidence of Be stars in massive clusters (Bastian et al. 2017) and the apparent bimodal spin distribution, which may require binary and/or environmental effects. All of this evidence may point to a large fraction of Be stars arising from binary channels in which the Be progenitor accreted mass/AM from a companion star. Indeed, binary models of Be star formation (Pols et al. 1991; Shao \& Li 2014) can account for a large fraction of the Be star population. de Mink et al. (2013) showed that the fraction of rapidly rotating postmass transfer systems expected from binary population synthesis is similar to the observed number of Be stars. Our binary models in Section 3.5 show that Be star formation is certainly promoted by mass accretion, either via stable mass transfer or stellar mergers. Hence, we agree that a substantial fraction of Be stars arise from binary interactions, but we reaffirm that they can produced by single stars, especially in low-metallicity environments.

\subsection{The LBV phenomenon}

An implication of our work is that centrifugally driven mass-loss could be related to LBV outbursts, especially S Doradus variations. Indeed, the centrifugal mass-loss rates we predict are similar to LBV mass-loss rates of a few $10^{-5} \mathrm{M}_{\odot} \mathrm{yr}^{-1}$ during $\mathrm{S}$ Doradus outbursts (Smith 2014, 2017). Intriguingly, we predict the highest mass-loss rates for stars near the S Doradus instability region (Fig. 3), because this is when the cores of very massive stars are contracting rapidly and providing AM to the surface layers, driving rapid centrifugal mass-loss. While measuring the rotation rates of LBVs is difficult, 
Groh, Hillier \& Damineli (2006) and Groh et al. (2009) have indeed demonstrated that some LBVs exhibit very rapid rotation, near their break-up rates, consistent with our suggestion of centrifugally driven outbursts.

We note that our models predict post-MS centrifugally driven mass-loss to be most common for solar metallicity at masses of $\sim 15-25 \mathrm{M}_{\odot}$, or luminosities of $\sim 10^{5} \mathrm{~L}_{\odot}$, near or below the bottom of the LBV region shown in Fig. 5. In light of the lower masses and luminosities recently measured for some LBVs (Smith \& Tombleson 2015; Smith 2019; Smith et al. 2019), the instability region should be revised downward. Since our models do not rely on super-Eddington mass-loss, these low-luminosity LBVs could naturally be explained by the centrifugal mass-loss processes we have investigated. Smith et al. (2019) also showed that some LBVs lie to the left of the classical S Doradus instability strip, a feature also seen in many of our models (e.g. Figs 7 and 11). Our models also predict high-luminosity LBVs to be more common in lowmetallicity environments where wind mass-loss is less important.

In this picture, LBVs may simply represent stars at the luminous end of a continuum of stars exhibiting centrifugal mass-loss. This continuum could extend from Be stars at luminosities of just $\sim 10^{2} \mathrm{~L}_{\odot}$, through $\operatorname{sgB}[\mathrm{e}]$ stars with luminosities of $\sim 10^{5} \mathrm{~L}_{\odot}$, all the way to LBVs with $L \gtrsim 10^{6} \mathrm{~L}_{\odot}$. The observational characteristics may be quite different, especially since the massive end of this continuum has near-Eddington luminosities, while radiative acceleration is negligible for ordinary Be stars. Hence, we may not expect to see centrifugally expelled decretion discs around LBVs like we do in Be stars, because such discs would quickly blown away by radiation force, or they would collapse due to 'Poynting-Robertson' drag (Owocki et al. 1998) on lines of irongroup elements.

The centrifugal mass-loss and/or outburst process may also be quite different in stars of different types. In MS Be stars, the iron opacity peak is relatively near the photosphere, where the overlying mass is small ( $\lesssim 10^{-5} \mathrm{M}_{\odot}$, Fig. 13) and the thermal time is small, promoting shorter and less violent (or non-existent) outbursts. In contrast, for post-MS LBV stars, the mass of the iron opacity peak can be much deeper, where the overlying mass is larger $\left(\sim 10^{-2} \mathrm{M}_{\odot}\right)$ and the thermal time is large, promoting longer and more violent outbursts. Outbursts of more massive stars may also be more common and more violent due to the role of radiation pressure, though a detailed prediction of centrifugally driven outbursts in LBVs awaits future work. For now, it remains difficult to explain the giant eruptions of LBVs like $\eta$ Carina, which can expel more than $10 \mathrm{M}_{\odot}$ of material (Smith et al. 2003; Smith \& Ferland 2007). While the iron opacity peak can extend to such depths in yellow/red supergiants, this occurs when the surface convection zone becomes very deep, the star's moment of inertia and spin period increases rapidly, and centrifugally driven mass-loss is not expected to occur.

\subsection{Case B stellar mergers}

In Section 3.5, we showed how case A mass accretion near the end of the MS (as could result from a stellar merger) can intensify centrifugal mass-loss when the star evolves off the MS. It would also be interesting to investigate case B mass accretion that can occur when the star crosses the Hertzsprung gap and can swallow a small companion. Justham, Podsiadlowski \& Vink (2014) performs a detailed investigation of the evolution of such merger products (not including updated AM transport prescriptions). Unlike our case A merger products that resemble single stars of increased mass that usually evolve into red supergiants, case B mass accretion produces stars that typically spend the rest of their lives as blue/yellow supergiants residing near the LBV instability strip. These stars do not evolve into red supergiants with large moments of inertia, and their cores continue to contract as they evolve. Hence, they could potentially exhibit centrifugal mass-loss throughout helium-burning until core-collapse. Centrifugal mass-loss from such stars could produce some LBV stars, and it could contribute to pre-SN outbursts of type IIn progenitor stars, some of which are known to resemble LBVs (e.g. Gal-Yam \& Leonard 2009; Smith et al. 2010; Mauerhan et al. 2013; Ofek et al. 2013, 2014; Smith, Mauerhan \& Prieto 2014; Smith 2014; Elias-Rosa et al. 2016; Tartaglia et al. 2016).

\subsection{Comparison with observed rotational velocities}

It is useful to compare our predictions with observed rotation rates of evolving massive stars, and with prior stellar models. Several studies (Huang \& Gies 2006a; Hunter et al. 2008; Dufton et al. 2013) have indicated a range of initial rotation rates for MS massive stars, with typical rotation rates in the range of $0-250 \mathrm{~km} \mathrm{~s}^{-1}$, and a small tail extending to higher velocities. Our models with $200 \mathrm{~km} \mathrm{~s}^{-1}$ are thus fairly typical massive stars, or perhaps slightly more rapidly rotating than average.

Measurements of post-MS rotation rates are more sparse but very informative, and have been made both in the LMC and SMC (Dufton et al. 2006a; McEvoy et al. 2015) and in the Galaxy (Fukuda 1982; Verdugo, Talavera \& Gómez de Castro 1999; Abt, Levato \& Grosso 2002; Abt 2003; Huang \& Gies 2006b). These works find fairly small rotational velocities for most A/B-type supergiant stars (which presumably have just evolved off the MS), with many in the range of $0-100 \mathrm{~km} \mathrm{~s}^{-1}$, and decreasing rotation towards lower temperature/gravity. For relatively low-mass (5-15 $\mathrm{M}_{\odot}$ ) sub-giants, nearly solid body rotation and nearly constant rotational velocity on the MS appears to match the data fairly well (Abt et al. 2002; Huang et al. 2010). Agreement with our models and others (e.g. Meynet \& Maeder 2000; Heger, Langer \& Woosley 2000; Ekström et al. 2008) is fairly good on the MS where they predict smaller amounts of differential rotation. However, our modelled rotational evolutions (Figs 4 and 6) predict slightly faster rotation than typically observed after the TAMS. In post-MS stars, the surface rotational velocity falls faster than expected from solid-body rotation (Verdugo et al. 1999; Abt 2003), and is below the predictions of our models. While our models do develop differential rotation after the TAMS, the level of differential rotation is less than most other models.

Possible explanations for these discrepancies are that our AM transport prescription predicts too much core-envelope coupling, or that our wind prescription predicts too little mass-loss. However, the works presented above point out that models with weaker AM transport and stronger winds (e.g. Meynet \& Maeder 2000, 2003) also predict rotation rates somewhat larger than observed in many cases. So, the source of the discrepancy is unclear. Some blue supergiants could be helium-burning stars on a blue loop that could have spun down via mass-loss during a red supergiant phase (though blue loops can also cause fast rotation if the star did not lose much AM as a supergiant; Heger \& Langer 1998). It has also been pointed out (Townsend, Owocki \& Howarth 2004) that in stars with significant centrifugal distortion, the spectrum is dominated by polar regions (due to gravity darkening near the equator) where the rotational velocity is small, so observed rotational velocities do not represent surface-averaged rotation speeds. Lastly, many of the studies discussed above do not include Be stars, which are the fastest rotating stars, and so those studies are biased towards the slowly rotating end of the distribution. 
A final important possibility to consider is that convective regions do not rotate rigidly as assumed in nearly all stellar evolution codes, but instead exhibit strong differential rotation as suggested by Kissin \& Thompson (2015). Massive post-MS stars begin to develop deep convective envelopes due to helium/iron opacity peaks for surface temperatures smaller than $\sim 20000 \mathrm{~K}$, or even higher temperatures for stars near the Eddington limit. If these convective zones rotate differentially, the surface rotation rate can be slower than the rotation rate at the top of the radiative region. In this case, modelled surface rotation rates may better agree with data and may remain smaller than the surface breakup rate. The internal rotation rate, however, could still exceed the break-up rate, and centrifugal mass-loss could occur via instabilities as discussed in Section 4. This could help explain why post-MS mass-loss sometimes occurs via large eruptions emanating from deeper layers of the star, in contrast to the small outbursts typical of Be stars apparently originating near the surface. We hope to explore this possibility in future work.

\subsection{Model uncertanities}

A significant uncertainty in our analysis is the structure of the outer layers of very luminous models, where convection becomes inefficient and the flux is locally super-Eddington due to iron and helium opacity bumps. Our models use MESA's MLT ++ prescription for handling these layers (Paxton et al. 2013), resulting in a more compact envelope than models without a similar prescription (e.g. Sanyal et al. 2017). Neither set of models is perfect, and radiation hydrodynamics simulations (Jiang et al. 2015, 2017, 2018) may be needed to guide the construction of better models. In any case, we suspect centrifugal mass-loss will be more important if stars develop inflated envelopes. Since these envelopes have very low masses but large radial extents (especially at high metallicity), they decrease the star's rotational break-up rate without significantly increasing the star's moment of inertia. Hence, the maximum AM content $J_{\mathrm{c}}$ of such stars may be smaller than our models, making them more susceptible to centrifugal mass-loss.

A second uncertainty is that of line-driven wind mass-loss rates. For hot stars, we adopt the widely used Vink prescription (Vink, de Koter \& Lamers 2001), but reduced by a factor of 2 . If the appropriate reduction factor is even larger, as advocated in many recent works (see review in Smith 2014), then stars will lose less AM on the MS and centrifugal mass-loss will become more important. The main effect of less efficient winds is similar to that of lower metallicity: an upward shift in the maximum stellar mass/luminosity to which centrifgual mass-loss extends.

\section{CONCLUSION}

We have examined the rotational evolution of massive stars, examining whether their outer layers can spin fast enough to centrifugally expel matter. Unlike most prior analyses (e.g. Ekström et al. 2008; Hastings et al. 2019), we incorporate very efficient angular momentum (AM) transport within our stellar models as required by asteroseismic observations (e.g. Mosser et al. 2012; Cantiello et al. 2014; Gehan et al. 2018; Fuller et al. 2019). Additionally, we extend our models past the main sequence to investigate post-mainsequence centrifugal mass-loss. We find that efficient AM transport from the contracting core to the expanding envelope can sometimes spin-up the surface layers to break up, necessitating centrifugal mass-loss. Centrifugal mass-loss is most prolific near the end of the main sequence and the early post-main sequence as massive stars cross the Hertzsprung gap. During these phases of evolution, the expanding envelope contains very little mass and the core contracts rapidly, donating enough AM to spin-up surface layers towards break-up velocities.

At solar metallicity, this effect is strongest for $\sim 20 \mathrm{M}_{\odot}$ stars (Fig. 5), with some dependence on wind mass-loss rates. Lower mass stars have smaller core masses/radii such that the core's contraction is not sufficient to spin-up outer layers. Higher mass stars lose most of their AM via line-driven winds, preventing centrifugal mass-loss. At low metallicity, the line-driven massloss weakens, promoting centrifugal mass-loss, especially in more massive stars (Fig. 7). While centrifugal mass-loss may generate circumstellar 'decretion' discs and could drive outbursts, it cannot expel the entire hydrogen envelope, and radiatively driven winds or binary interactions are still responsible for the bulk of massloss. We confirm that some Be stars can form from rapidly rotating single stars due to outward AM transport within the star. However, in many stars, accretion from a binary companion is likely crucial for supplying enough AM to drive centrifugal mass-loss. Hence, both effectively single and binary stellar evolution may contribute substantially to the Be star phenomenon. Interestingly, stars that accrete a small amount of mass $\left(\sim 1 \mathrm{M}_{\odot}\right)$ near the end of the MS are most prone to post-MS centrifugal mass-loss (Fig. 11), because the merger spins them up without significant extending the MS lifetime and hence the AM loss due to line-driven winds.

We predict the largest centrifugal mass-loss rates $(\sim$ $10^{-4} \mathrm{M}_{\odot} \mathrm{yr}^{-1}$ ) for massive post-main-sequence stars near the S Doradus instability strip. Hence, we posit that $S$ Doradus variations and outbursts from some LBV stars are driven by centrifugal mass-loss, in agreement with the apparent rapid rotation of some LBVs (Groh et al. 2006, 2009). Our prediction of extreme centrifugal mass-loss extending down to masses of $\sim 20 \mathrm{M}_{\odot}$ is similar to the lower end of LBVs observed in the Milky Way (Smith et al. 2019). However, the absence of such low-luminosity LBVs in the Magellanic clouds suggest metallicity may also be an important factor in driving LBV outbursts, perhaps due to a radiation-centrifugal instability (Section 4). Preliminary analytical and numerical calculations indicate a centrifugal instability could operate in massive stars crossing the Hertzspung gap, driven by AM transport into outwardly perturbed fluid elements. The instability can originate deep in the star (at the iron or helium opacity bumps) and could potentially drive outbursts that partially expel overlying layers. We hope to examine this instability and its relation to LBV outbursts in future work.

\section{ACKNOWLEDGEMENTS}

We thank Nathan Smith and Matteo Cantiello for very helpful suggestions. This research is funded in part by an Innovator Grant from The Rose Hills Foundation, and the Sloan Foundation through grant no. FG-2018-10515.

\section{REFERENCES}

Abt H. A., 2003, ApJ, 582, 420

Abt H. A., Levato H., Grosso M., 2002, ApJ, 573, 359

Aerts C., Lamers H. J. G. L. M., Molenberghs G., 2004, A\&A, 418, 639

Aidelman Y., Cidale L. S., Zorec J., Panei J. A., 2018, A\&A, 610, A30

Bastian N. et al., 2017, MNRAS, 465, 4795

Cantiello M., Mankovich C., Bildsten L., Christensen-Dalsgaard J., Paxton B., 2014, ApJ, 788, 93

Castor J. I., Abbott D. C., Klein R. I., 1975, ApJ, 195, 157

de Mink S. E., Langer N., Izzard R. G., Sana H., de Koter A., 2013, ApJ, 764, 166

Deheuvels S. et al., 2014, A\&A, 564, A27 
Dufton P. L., Ryans R. S. I., Simón-Díaz S., Trundle C., Lennon D. J., 2006a, A\&A, 451, 603

Dufton P. L. et al., 2006b, A\&A, 457, 265

Dufton P. L. et al., 2013, A\&A, 550, A109

Dwarkadas V. V., Owocki S. P., 2002, ApJ, 581, 1337

Eggenberger P. et al., 2017, A\&A, 599, A18

Eggenberger P., den Hartogh J. W., Buldgen G., Meynet G., Salmon S. J. A. J., Deheuvels S., 2019, A\&A, 631, L6

Ekström S., Meynet G., Maeder A., Barblan F., 2008, A\&A, 478, 467

Elias-Rosa N. et al., 2016, MNRAS, 463, 3894

Friend D. B., Abbott D. C., 1986, ApJ, 311, 701

Fukuda I., 1982, PASP, 94, 271

Fuller J., Piro A. L., Jermyn A. S., 2019, MNRAS, 485, 3661

Gagnier D., Rieutord M., Charbonnel C., Putigny B., Espinosa Lara F., 2019, A\&A, 625, A88

Gal-Yam A., Leonard D. C., 2009, Nature, 458, 865

Gehan C., Mosser B., Michel E., Samadi R., Kallinger T., 2018, A\&A, 616, A24

Georgy C., Ekström S., Granada A., Meynet G., Mowlavi N., Eggenberger P., Maeder A., 2013, A\&A, 553, A24

Glatzel W., 1998, A\&A, 339, L5

Gräfener G., Owocki S. P., Vink J. S., 2012, A\&A, 538, A40

Granada A., Ekström S., Georgy C., Krtička J., Owocki S., Meynet G., Maeder A., 2013, A\&A, 553, A25

Groh J. H., Hillier D. J., Damineli A., 2006, ApJ, 638, L33

Groh J. H. et al., 2009, ApJ, 705, L25

Hastings B., Wang C., Langer N., 2019, A\&A, 633, A165

Heger A., Langer N., 1998, A\&A, 334, 210

Heger A., Langer N., Woosley S. E., 2000, ApJ, 528, 368

Hirschi R., et al., 2006, Rev. Mod. Astron., 19, 101

Huang W., Gies D. R., 2006a, ApJ, 648, 580

Huang W., Gies D. R., 2006b, ApJ, 648, 591

Huang W., Gies D. R., McSwain M. V., 2010, ApJ, 722, 605

Humphreys R. M., Davidson K., 1994, PASP, 106, 1025

Hunter I., Lennon D. J., Dufton P. L., Trundle C., Simón-Díaz S., Smartt S. J., Ryans R. S. I., Evans C. J., 2008, A\&A, 479, 541

Jiang Y.-F., Cantiello M., Bildsten L., Quataert E., Blaes O., 2015, ApJ, 813, 74

Jiang Y.-F., Cantiello M., Bildsten L., Quataert E., Blaes O., 2017, ApJ, 843, 68

Jiang Y.-F., Cantiello M., Bildsten L., Quataert E., Blaes O., Stone J., 2018, Nature, 561, 498

Justham S., Podsiadlowski P., Vink J. S., 2014, ApJ, 796, 121

Keller S. C., Bessell M. S., Da Costa G. S., 2000, AJ, 119, 1748

Kissin Y., Thompson C., 2015, ApJ, 808, 35

Kraus M., 2019, Galaxies, 7, 83

Langer N., 1998, A\&A, 329, 551

Langer N., García-Segura G., Mac Low M.-M., 1999, ApJ, 520, L49

Ledoux P., 1945, ApJ, 102, 143

Maeder A., 1999, A\&A, 347, 185

Maeder A., Meynet G., 2000, ARA\&A, 38, 143

Maeder A., Meynet G., 2000, A\&A, 361, 159

Maeder A., Meynet G., 2001, A\&A, 373, 555

Maeder A., Meynet G., 2012, Rev. Mod. Phys., 84, 25

Mauerhan J. C. et al., 2013, MNRAS, 430, 1801

McEvoy C. M. et al., 2015, A\&A, 575, A70

Meynet G., Maeder A., 2000, A\&A, 361, 101

Meynet G., Maeder A., 2003, A\&A, 404, 975

Mokiem M. R. et al., 2006, A\&A, 456, 1131

Mosser B. et al., 2012, A\&A, 548, A10

Müller P. E., Vink J. S., 2014, A\&A, 564, A57

Ofek E. O. et al., 2013, Nature, 494, 65

Ofek E. O. et al., 2014, ApJ, 789, 104

Owocki S., 2014, preprint (arXiv:1409.2084)

Owocki S. P., 2015, in Vink J. S., ed., Astrophysics and Space Science

Library, Vol. 412, Very Massive Stars in the Local Universe, Springer, Germany, p. 113

Owocki S. P., Cranmer S. R., Gayley K. G., 1998, Ap\&SS, 260, 149
Paxton B., Bildsten L., Dotter A., Herwig F., Lesaffre P., Timmes F., 2011, ApJS, 192, 3

Paxton B. et al., 2013, ApJS, 208, 4

Paxton B. et al., 2015, ApJS, 220, 15

Paxton B. et al., 2018, ApJS, 234, 34

Paxton B. et al., 2019, preprint (arXiv:1903.01426)

Peters M. et al., 2019, preprint (arXiv:1912.05627)

Pols O. R., Cote J., Waters L. B. F. M., Heise J., 1991, A\&A, 241, 419

Ramírez-Agudelo O. H. et al., 2013, A\&A, 560, A29

Rivinius T., Carciofi A. C., Martayan C., 2013, A\&AR, 21, 69

Sanyal D., Langer N., Szécsi D., Yoon S.-C., Grassitelli L., 2017, A\&A, 597, A71

Shao Y., Li X.-D., 2014, ApJ, 796, 37

Smith N., 2014, ARA\&A, 52, 487

Smith N., 2017, Phil. Trans. R. Soc. London Ser. A, 375, 20160268

Smith N., 2019, MNRAS, 489, 4378

Smith N., Ferland G. J., 2007, ApJ, 655, 911

Smith N., Tombleson R., 2015, MNRAS, 447, 598

Smith N., Gehrz R. D., Hinz P. M., Hoffmann W. F., Hora J. L., Mamajek

E. E., Meyer M. R., 2003, AJ, 125, 1458

Smith N. et al., 2010, AJ, 139, 1451

Smith N., Mauerhan J. C., Prieto J. L., 2014, MNRAS, 438, 1191

Smith N., Aghakhanloo M., Murphy J. W., Drout M. R., Stassun K. G., Groh J. H., 2019, MNRAS, 488, 1760

Spruit H. C., 2002, A\&A, 381, 923

Tartaglia L. et al., 2016, MNRAS, 459, 1039

Tassoul J.-L., 1978, Theory of Rotating Stars, Princeton University Press, USA

Townsend R. H. D., Owocki S. P., Howarth I. D., 2004, MNRAS, 350, 189

Van Reeth T. et al., 2018, A\&A, 618, A24

Verdugo E., Talavera A., Gómez de Castro A. I., 1999, A\&A, 346, 819

Vink J. S., 2017, Phil. Trans. R. Soc. London Ser. A, 375, 20160269

Vink J. S., Gräfener G., 2012, ApJ, 751, L34

Vink J. S., de Koter A., Lamers H. J. G. L. M., 2001, A\&A, 369, 574

von Zeipel H., 1924, MNRAS, 84, 665

Zahn J.-P., 1992, A\&A, 265, 115

Zorec J. et al., 2016, A\&A, 595, A132

\section{APPENDIX A: NEGLECT OF CORIOLIS FOR CE}

We have ignored the Coriolis force in equation (26), which may not always be appropriate. However, we note that Ledoux (1945) eliminated the Coriolis forces by considering a frame that changes rotation rate throughout the pulsation cycle. In principle, such a frame could be used in our analysis as well.

Inclusion of the Coriolis force would add a term $-2 i \Omega \omega \rho \xi_{\phi} \sin \theta$ to the right-hand side, where $\xi_{\phi}$ is the displacement in the $\phi$ direction. The Coriolis force can thus be neglected if $\omega^{2} \Delta r \gtrsim$ $\omega \Omega \xi_{\phi}$. One could imagine strong torques (due to magnetic tension forces or convective Reynold's stresses) that overwhelm the Coriolis force and prevent fluid elements from moving horizontally and satisfying this approximation. To see this, consider the effect of a radial magnetic field. The $\phi$ component of the momentum equation (assuming axisymmetry) is

$$
-\omega^{2} \xi_{\phi} \sim 2 i \omega \Omega\left(\xi_{\theta} \cos \theta-\Delta r \sin \theta\right)+\mathcal{O}\left(\omega_{\mathrm{A}}^{2} \xi_{\phi}\right) .
$$

The last term in equation (A1) represents magnetic tension forces, assuming the magnetic fields and horizontal displacements vary on a length scale of $r$. For convective torques, the Alfvén frequency $\omega_{A}=$ $B_{r} / \sqrt{4 \pi \rho r^{2}}$ should be replaced by a convective turnover frequency $\omega_{\text {con }} v_{\text {con }} / r$. Hence, Coriolis forces can be ignored in this equation if $\omega_{\mathrm{A}}^{2} \xi_{\phi} \gtrsim \omega \Omega \Delta r$. Combining this requirement with that from the radial momentum equation, Coriolis forces are negligible if $\omega_{\mathrm{A}} \gtrsim \Omega$. In other words, torques due to magnetic fields or convection should 
be stronger than Coriolis torques to justify a spherical treatment of the problem.

Since the instability originates in the outer layers of stars, it is certainly possible that $\omega_{\mathrm{A}}$ or $\omega_{\text {con }}$ will be large enough to overwhelm Coriolis forces near the surface of the star. In these layers, $\omega_{\mathrm{A}}$ and $\omega_{\text {con }}$ become large as the density becomes small, whereas $\Omega$ is nearly constant. In the Sun, for example, both $\omega_{\mathrm{A}}$ and $\omega_{\text {con }}$ are a couple orders of magnitude larger than $\Omega$ near the surface. While both $\omega_{\mathrm{A}}$ and $\omega_{\text {con }}$ decrease deeper in the star, the shallow density gradient near the surfaces of massive stars (Fig. 13) helps them remain large. Additionally, for stars near the Eddington limit or near $\gamma=4 / 3$, the rotation rate $\Omega$ can be much less than break-up when the instability sets in, decreasing the Coriolis force. Thirdly, due to the very high luminosities and low densities of the envelopes, the convective velocities approach the sound speed deep in the star (e.g. near the iron opacity bump), increasing the magnetic/convective torques.

\section{APPENDIX B: MESA MODEL INLISTS}

We construct our stellar models using the MESA stellar evolution code (Paxton et al. 2011, 2013, 2015, 2018, 2019) version 11701.

The inlist is as follows:

\section{B1 MESA inlist}

\&star_job

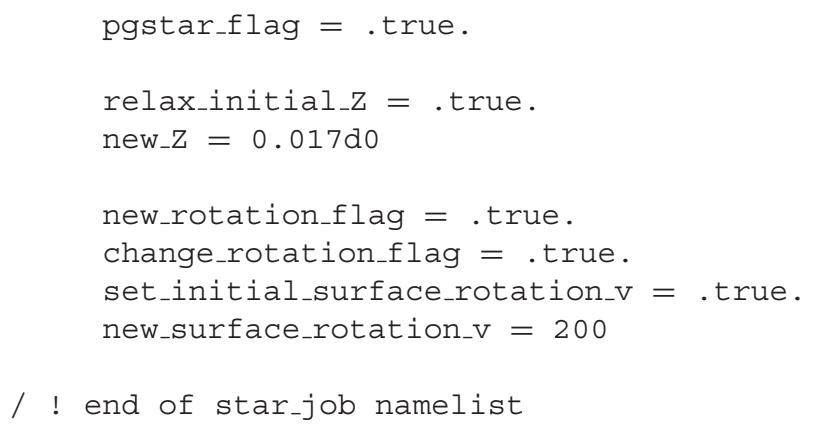

Convergence

okay_to_reduce_gradT_excess $=$.true. gradT_excess_age_fraction $=0.999 \mathrm{do}$ gradT_excess_max_change $=0.01 d 0$

timestep_factor_for_retries $=0.8$ timestep_factor_for_backups $=0.8$ min_timestep_factor $=0.9$

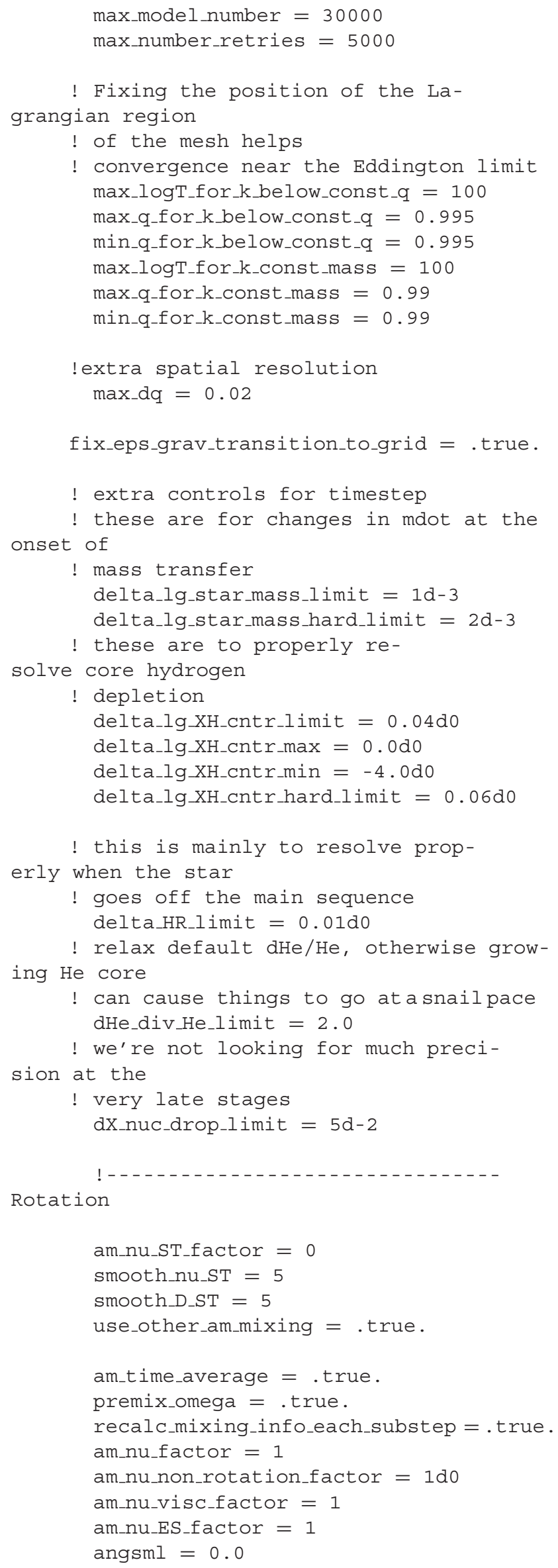


am_D_mix_factor $=3.33 \mathrm{~d}-2$

D_ES_factor $=1$

! this is to avoid odd be-

haviour when a star

! switches from accret-

ing to mass losing

max_mdot_jump_for_rotation $=1$ d99

MAIN

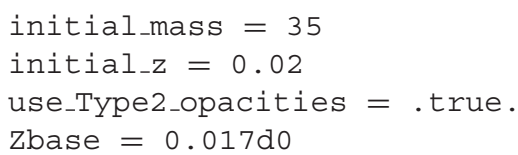

WIND

hot_wind_scheme = 'Dutch' cool_wind_RGB_scheme = 'Dutch' cool_wind_AGB_scheme = 'Dutch' RGB_to_AGB_wind_switch $=1 \mathrm{~d}-4$ Dutch_scaling_factor $=0.0 ! 0.5$ mdot_omega_power $=0.0 d 0$

! - $\ldots \ldots \ldots$ OVER-

SHOOTING

overshoot_f_above_nonburn_core $=0.025$ overshoot_f0_above_nonburn_core $=0.005$ overshoot_f_above_nonburn_shell $=0.025$ overshoot_f0_above_nonburn_shell $=0.005$ overshoot_f_below_nonburn_shell $=0.025$ overshoot_f0_below_nonburn_shell $=0.005$

overshoot_f_above_burn_h_core $=0.025$ overshoot_f0_above_burn_h_core $=0.005$ overshoot_f_above_burn_h_shell $=0.025$ overshoot_fo_above_burn_h_shell $=0.005$ overshoot_f_below_burn_h_shell $=0.025$ overshoot_f0_below_burn_h_shell $=0.005$

set_min_D_mix $=$.true.

min_D_mix $=1 d 2$

- MISC

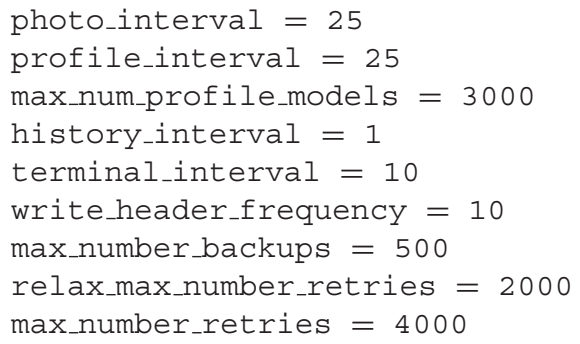

\section{B2 Implementation of mass-loss wind}

Our code in run_star_extras.f implementing mass-loss wind is as follows:

subroutine my_other_wind(id, Lsurf, Msurf, Rsurf, Tsurf, w, ierr) use star_def, only: star_info use star_lib, only: star_ptr integer, intent(in) : : id real (dp), intent(in) : : Lsurf, Msurf real (dp), intent(in) : : Rsurf, Tsurf !surface values (cgs)

real (dp), intent (out) : : w !wind in units of Msun/year (value

is $>0$

integer, intent(out) : : ierr

integer : : $\mathrm{y}$

!used for tanh smoothing

real (dp) : : I, r, r_old

!moment of inertia

!radius or the current model !radius of the previous model

real (dp) : : cen_wind, omega_crit lcentrifugal mass loss rate !critical rotational velocity

real (dp) :: Dutch_wind real (dp) : : w1, w2, alfa, temp ! used to calculate Dutch_wind

type (star_info), pointer : : s call star_ptr(id, s, ierr) if (ierr $/=0$ ) then ! OOPS return

end if

$\mathrm{w}=0$

ierr $=0$ 


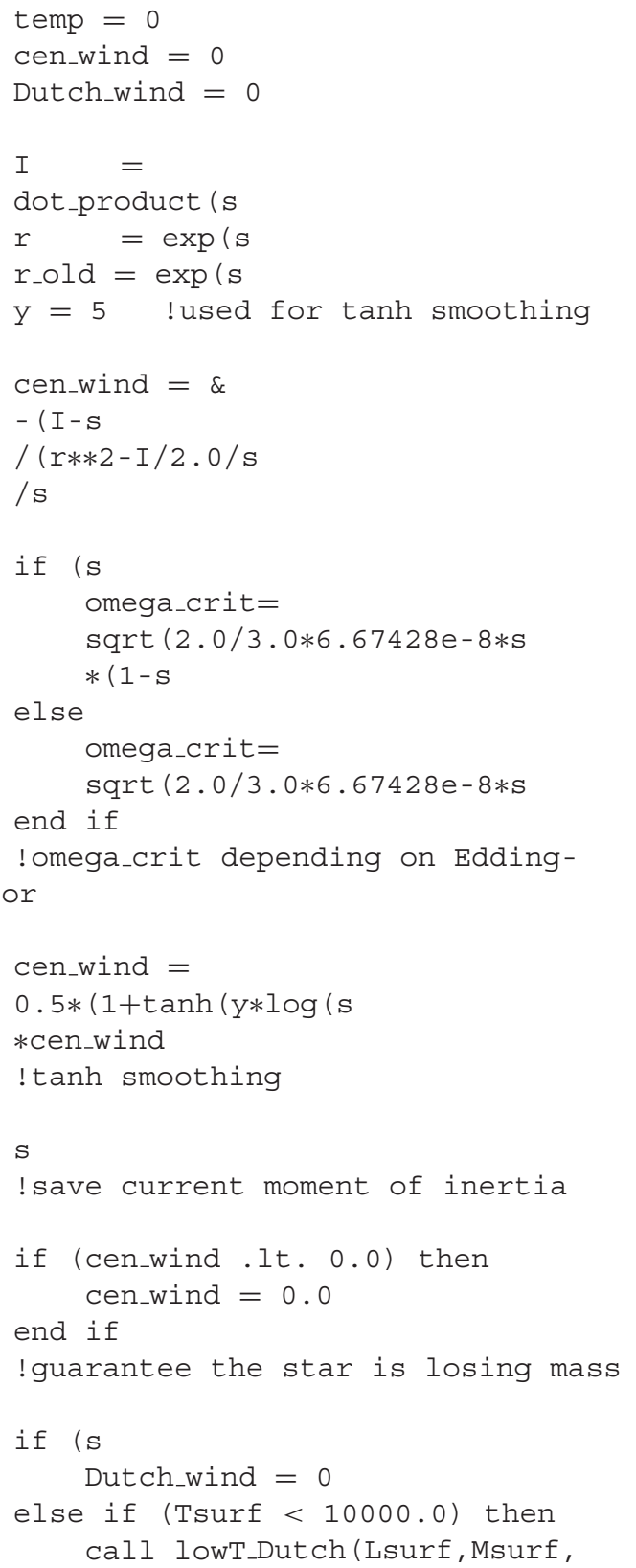

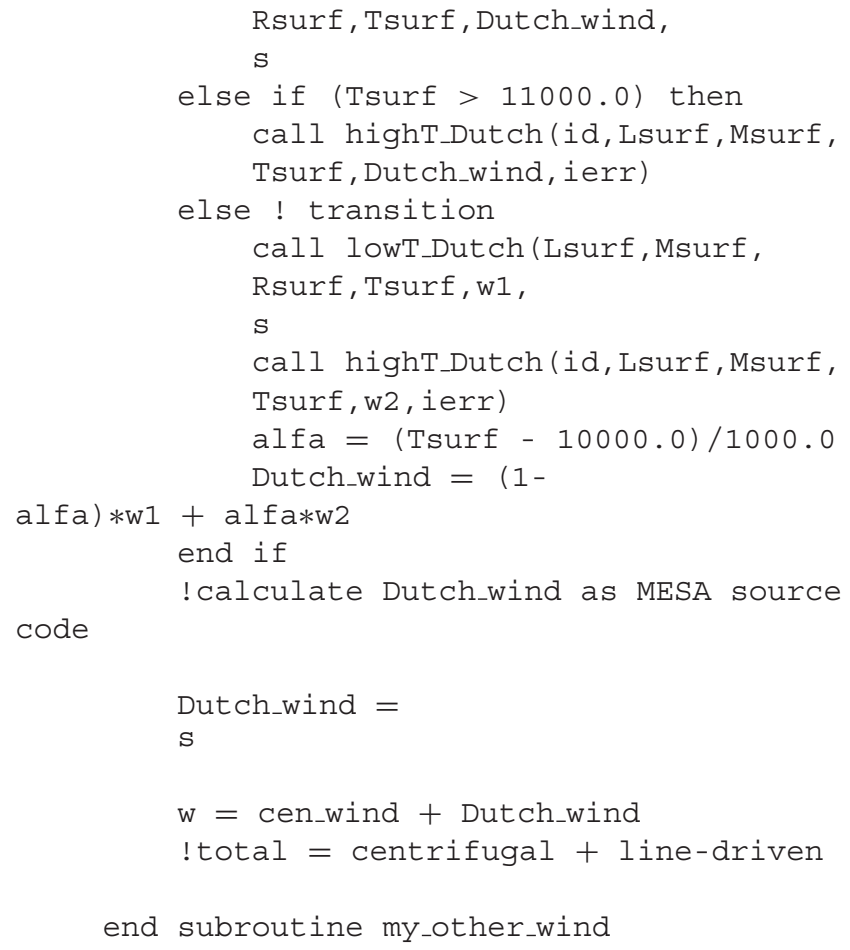

To run the my_other_wind routine, one must employ subroutines from MESA source code. They are eval_highT_Dutch, eval_lowT_Dutch, and other subroutines related to them. These subroutines are in the private/winds.f 90 file. Additionally, we include the TSF subroutine from Fuller et al. (2019) with minor modifications to implement angular momentum transport. We use a tanh function to smooth the transition of centrifugal mass-loss rate from 0 to a positive number when $\Omega>\Omega_{\text {crit }}$ as

$\dot{M}_{\text {cen }}=\frac{1}{2}\left(1+\tanh \left[\log \left(\frac{\Omega}{\Omega_{\text {crit }}}\right)^{y}\right]\right) \times \dot{M}$,

where $\dot{M}$ is calculated as in equation (14), and in our code $y=5$. This tanh smoothing represses numerical instabilities, and we find results with different values of $y$ are similar.

This paper has been typeset from a $\mathrm{T}_{\mathrm{E}} \mathrm{X} / \mathrm{L} \mathrm{T} \mathrm{T} \mathrm{X}$ file prepared by the author. 